\title{
Relación de agujeros negros súper masivos con propiedades globales de las galaxias que los albergan
}

\author{
Yvelice Soraya Castillo Rosales*
}

\section{RESUMEN}

Partiendo del trabajo de Tesis de Maestría de Castillo Rosales, Y. S., que incluyó una exhaustiva revisión bibliográfica de revistas profesionales de astronomía y astrofísica ("papers") que se refieren a agujeros negros súper masivos (en adelante, ANSs) y a varias propiedades de las galaxias que los albergan; se han seleccionado diez publicaciones que se consideraron representativas en cuanto a datos y a correlaciones significativas entre las masas de agujeros negros centrales y las propiedades globales de las galaxias anfitrionas. Tomando los datos de estas diez publicaciones, se seleccionaron 11 propiedades relevantes, que fueron agrupadas en tres tipos de tablas: galaxias elípticas, galaxias de disco y galaxias peculiares (peculiares incluye galaxias activas, irregulares y fusiones de galaxias). La muestra final es de 144 galaxias. En total se recopilaron 1,453 datos. Además se elaboraron nueve gráficos de correlaciones entre las masas de agujero negro súper masivo central de las galaxias de la muestra, con las nueve siguientes propiedades de las galaxias: dispersión central de velocidades, luminosidad del bulbo, radio de influencia del ANS, radio efectivo del bulbo, masa del esferoide, relación masaluminosidad total, corrimiento al rojo, distancia al centro de la Galaxia, y luminosidad en lejano infrarrojo. Con ayuda de los gráficos de correlaciones elaborados se hizo un análisis comparativo entre estas correlaciones y las correlaciones que arrojan cinco modelos teóricos recientes de formación y evolución de galaxias. Los resultados parecen apoyar las teorías de formación de galaxias a partir de halos de materia oscura, que posteriormente formarían galaxias de disco, cuyos agujeros negros centrales serían los motores del crecimiento galáctico, a tasas de acreción moderadas.

Palabras clave: agujero negro súper masivo (ANS), correlación, galaxia, dispersión de velocidades, evolución, formación.

\footnotetext{
* Yvelice Soraya Castillo Rosales, yvelicec@yahoo.com

Facultad de Ciencias Espaciales, Astronomía y Astrofísica, Observatorio Astronómico Centroamericano de Suyapa, Universidad Nacional Autónoma de Honduras.
} 


\section{ABSTRACT}

On the basis of Castillo, Y. S. Master Thesis, which include a comprehensive bibliographic review from Astronomy and Astrophysics professional papers, related to super massive black holes (hereinafter SMBHs) and several properties of their host galaxies, ten publications considered representative has been selected, as regards data and significant correlations between central black hole masses and the global properties of the host galaxies. Taking these ten publications data, have been selected 11 relevant properties that were grouped into three kinds of tables: elliptical galaxies, disk galaxies and peculiar galaxies (peculiar includes active, irregular galaxies and mergers of galaxies). The final sample is 144 galaxies. 1,453 Data were collected in total. Also developed nine plots of correlations between central super massive black hole masses from the sample versus the following nine properties of galaxies: central velocity dispersion, bulb Luminosity, SMBH influence radio, bulb effective radio, spheroid mass, mass-luminosity ratio, redshift, distance from the center of the Galaxy, and far infrared luminosity. With the help of correlation plots became a comparative analysis between these correlations and correlations from five recent theoretical models of formation and evolution of galaxies. The results seem to support the formation of galaxies from dark matter halos that would later form disk galaxies, whose central black holes would be galactic growth engines, at moderate accretion rates.

Keywords: super massive black hole (SMBH), correlation, galaxy, velocity dispersion, evolution, formation. 


\section{INTRODUCCIÓN}

Desde hace algunas décadas, y más especialmente desde el lanzamiento del telescopio espacial Hubble, muchos investigadores han dedicado grandes esfuerzos para escudriñar las regiones más interiores de las galaxias. Esto se da gracias a que la resolución espacial de los telescopios más recientes ha mejorado significativamente, facilitando los estudios de esas regiones. A principios de los años noventa captó gran interés la búsqueda de agujeros negros súper masivos (ANSs) en el centro de algunas galaxias; pero una vez que se comprobó su presencia en los núcleos de una gran cantidad de galaxias, el interés se desvió luego a determinar las correlaciones que existen entre las masas de ANSs y algunas propiedades globales de las galaxias que los albergan, con dos intenciones principales: a) hacer más fácil la medición de las masas de ANSs, y b) analizar qué injerencia tienen estas relaciones en la historia evolutiva de los ANSs o en la historia evolutiva de la estructura galáctica en general.

Actualmente no se ha logrado llegar a un acuerdo sobre este segundo punto, pues todavía no se ha logrado comprobar a ciencia cierta cuál es el proceso exacto que ha causado la formación y el crecimiento de losANSs y de las galaxias en general.

En un esfuerzo por determinar cuál de los modelos actuales de formación y de crecimiento de agujeros negros súper masivos se ajusta mejor a las propiedades de agujeros negros, medidas en base a observaciones y cuáles son los parámetros más relevantes que intervienen en los procesos evolutivos de los ANSs, se hizo un análisis comparativo de los datos que arrojan varios modelos de crecimiento de ANSs, con los datos observacionales y las correlaciones de propiedades obtenidas por varios grupos de investigación, para dilucidar cuál de los modelos propuestos se adapta mejor a los datos observacionales.

\section{OBJETIVOS}

\section{Objetivo General}

Revisar las correlaciones más relevantes, estudiadas en el proyecto de Tesis de Castillo Rosales, Y. S., ahora con una muestra de más galaxias, espirales, elípticas y peculiares.

\section{Objetivo Específico}

Comparar las correlaciones más confiables de masas de agujeros negros súper 
masivos, con otras propiedades de las galaxias anfitrionas, con los resultados de modelos recientes de crecimiento y evolución de agujeros negros súper masivos.

\section{MÉTODO}

Una vez recopilados muchos valores de masas de agujeros negros súper masivos centrales y de propiedades de sus galaxias anfitrionas publicados en artículos de revistas especializadas, por los mejores grupos de investigación en agujeros negros súper masivos, estos valores se asociaron como correlaciones entre las masas de agujeros negros centrales y varias de las propiedades recopiladas. Estas correlaciones basadas en datos observacionales fueron comparadas con las correlaciones que arrojan cinco modelos teóricos de formación y evolución de galaxias, publicados en el año 2008.

Como fuente de datos para obtener propiedades de galaxias y masas de agujeros negros centrales se utilizaron las revistas astronómicas especializadas de $\mathrm{ADS}^{1}$, ArXiv $^{2}$, HYPERLEDA $^{3}$, Rc3 $^{4}$, NED $^{5}$ y Vizier ${ }^{6}$. La muestra seleccionada incluye 53 galaxias espirales, 51 galaxias elípticas y 40 galaxias peculiares.

La propiedades que se seleccionaron para la muestra son 11: (1) corrimiento al rojo (z), (2) distancia a la galaxia, (3) tipo del núcleo, (4) masa calculada del agujero negro súper masivo central, (5) radio de influencia del ANS, (6) dispersión central de velocidades, (7) radio efectivo del bulbo, (8) luminosidad en lejano infrarrojo, (9) luminosidad del bulbo, (10) masa del bulbo, (11) relación masa-luminosidad de toda la galaxia. Aunque se buscaron las 11 propiedades para 144 galaxias, no se lograron obtener para todas, puesto que las galaxias peculiares aún no han sido suficientemente estudiadas. Se obtuvieron desde 3 hasta 11 datos por galaxia, con un total de 1,453 datos.

\footnotetext{
${ }^{1}$ Base de datos astrofísicos de la NASA ("NASA Astrophysics Data System"), Http://adsabs.harvard.edu/abstract service.html

2 Base de datos de artículos remitidos a revistas, sin haber sido publicados ("pre-prints"), http://arxiv.org/find/astro-ph

${ }^{3}$ Base de datos del Observatorio de Lyon-Meudon (Lyon-Meudon Extragalactic Database), http://leda.univ-lyon1.fr/search.html

"Tercera Referencia del Catálogo de Galaxias Brillantes", ("The Third Reference Catalogue of Bright Galaxies"), http://cadcwww.dao.nrc.ca/astrocat/rc3.html

${ }^{5}$ Base de Datos Extragalácticos de la NASA (NASA Extragalactic Database), http://nedwww.ipac.caltech.edul http://vizier.u-strasbg.fr/viz-bin/VizieR
} 
Los datos se estandarizaron, considerando una constante de Hubble para la expansión del universo de $70 \mathrm{~km} / \mathrm{s} / \mathrm{Mpc}$. También se hicieron las conversiones necesarias para que todos los datos puedan compararse en las mismas unidades.

Con los datos recopilados se elaboraron 3 tablas, una para cada tipo de galaxia, para comparar visualmente las propiedades de unas galaxias con otras. Los datos se ingresaron en una hoja electrónica de Excell para facilitar su manipulación, análisis y la elaboración de gráficos.

Se elaboraron nueve gráficos de correlaciones entre las masas de ANSs centrales y las nueve propiedades globales más significativas de las galaxias. Se decidió no emplear la muestra de galaxias peculiares para la elaboración de gráficos de correlaciones, porque para éstas sólo se encontraron distancias, masas de agujeros negros centrales y luminosidades en lejano infrarrojo. Al final se enumeran los resultados obtenidos y las conclusiones respectivas.

\section{RESULTADOS}

\section{Tablas}

Tres tablas de datos, que muestran entre 3 y 11 propiedades para cada una de 144 galaxias. Se agruparon las galaxias según tipos espiral, elíptico y peculiar.

\section{Gráficos}

Hay nueve gráficos que presentan las correlaciones entre la masa del agujero negro súper masivo central de cada galaxia contra nueve propiedades, en orden de importancia: (1) dispersión central de velocidades, en km/s; (2) luminosidad del bulbo, en luminosidades solares; (3) masa del esferoide o bulbo, en masas solares; (4) radio efectivo del bulbo, en kpc; (5) corrimiento al rojo (z); (6) distancia al centro de nuestra Galaxia, en Mpc; (7) relación masa-luminosidad de la galaxia, en masas y luminosidades solares; (8) radio de influencia del ANS, en segundos de arco; (9) luminosidad en lejano infrarrojo, en luminosidades solares. 


\begin{tabular}{|c|c|c|c|c|c|c|c|c|c|c|c|}
\hline \multicolumn{12}{|c|}{ TABLA 1: GALAXIAS ELIPTICAS (columnas 1 a 12) } \\
\hline No. & Nombre & $\begin{array}{c}\text { Tipo } \\
\text { Hubble }\end{array}$ & $\begin{array}{c}Z \\
\text { Estimado } \\
\text { con } \\
\mathrm{Ho}=70 \\
\mathrm{Km} / \mathrm{s} / \mathrm{mpc}\end{array}$ & $\begin{array}{l}\text { D, Mpc } \\
(\mathrm{H} 0=70)\end{array}$ & $\begin{array}{c}\text { Tipo } \\
\text { nuclear }\end{array}$ & $\begin{array}{l}\mathrm{M}_{\mathrm{BH}}, \\
\text { Msol }\end{array}$ & $\begin{array}{l}\text { Error } \\
\mathrm{M}_{\mathrm{BH}}{ }^{-}\end{array}$ & $\begin{array}{l}\text { Error } \\
\mathrm{M}_{\mathrm{BH}}+\end{array}$ & Ref & $\left|\begin{array}{c}\mathrm{R}_{\mathrm{BH}} \\
\operatorname{arcsec}\end{array}\right|$ & Nres \\
\hline (1) & $(2)$ & (3) & $(4)$ & $(5)$ & (6) & (7) & (8) & (9) & $(10)$ & (11) & $(12)$ \\
\hline 1 & 221 & cE2 & 0.00019 & 0.81143 & & $\begin{array}{l}3.90 \mathrm{E} \\
+06\end{array}$ & $\begin{array}{l}9.00 \mathrm{E} \\
+05\end{array}$ & $\begin{array}{l}9.00 \mathrm{E} \\
+05\end{array}$ & S & & \\
\hline 2 & 821 & E4p & 0.006 & 24.1 & & $\begin{array}{l}3.70 \mathrm{E} \\
+07\end{array}$ & $\begin{array}{l}8.00 \mathrm{E} \\
+06\end{array}$ & $\begin{array}{l}2.40 \mathrm{E} \\
+07\end{array}$ & S & 0.03 & 0.6 \\
\hline 3 & 1052 & $\overline{E 4}$ & 0.007 & 31.7143 & & $\begin{array}{l}1.95 \mathrm{E} \\
+08\end{array}$ & & & & & \\
\hline 4 & N4464 & & 0.004 & 16.9821 & & $\begin{array}{l}1.26 \mathrm{E} \\
+07 \\
\end{array}$ & & & & & \\
\hline 5 & 1692 & & 0.004 & 18.2786 & & $\begin{array}{l}1.00 \mathrm{E} \\
+08\end{array}$ & & & & & \\
\hline 6 & 1720 & & 0.004 & 17.4536 & & $\begin{array}{l}3.98 \mathrm{E} \\
+07\end{array}$ & & & & & \\
\hline 7 & 1883 & & 0.004 & 17.775 & & \begin{tabular}{|l}
$6.31 \mathrm{E}$ \\
+06 \\
\end{tabular} & & & & & \\
\hline 8 & 1913 & & 0.004 & 18.6214 & & $\begin{array}{l}3.16 \mathrm{E} \\
+06\end{array}$ & & & & & \\
\hline 9 & 2048 & & 0.004 & 17.6786 & & $\begin{array}{l}\begin{array}{l}2.00 \mathrm{E} \\
+06\end{array} \\
+0\end{array}$ & & & & & \\
\hline 10 & 2092 & & 0.004 & 17.2929 & & \begin{tabular}{|l|}
$1.58 \mathrm{E}$ \\
+08 \\
\end{tabular} & & & & & \\
\hline 11 & 2778 & E2 & 0.005 & 22.9 & & $\begin{array}{l}1.40 \mathrm{E} \\
+07\end{array}$ & $\begin{array}{l}9.00 \mathrm{E} \\
+06\end{array}$ & \begin{tabular}{|l|}
$\begin{array}{l}8.00 \mathrm{E} \\
+06\end{array}$ \\
\end{tabular} & $S$ & 0.02 & 0.4 \\
\hline 12 & 3377 & E5 & 0.003 & 11.2 & & $\begin{array}{l}1.00 \mathrm{E} \\
+08 \\
\end{array}$ & $\begin{array}{l}1.00 \mathrm{E} \\
+07 \\
\end{array}$ & \begin{tabular}{l|}
$9.00 E$ \\
+07 \\
\end{tabular} & S & 0.38 & 3.6 \\
\hline 13 & 3379 & E1 & 0.002 & 10.6 & & \begin{tabular}{|l|l|}
$1.00 \mathrm{E}$ \\
+08 \\
\end{tabular} & $\begin{array}{l}5.00 \mathrm{E} \\
+07 \\
\end{array}$ & \begin{tabular}{|l|}
$6.00 \mathrm{E}$ \\
+07 \\
\end{tabular} & $S$ & 0.2 & 1.9 \\
\hline
\end{tabular}

${ }^{8}$ (1) número asignado; (2) nombre más común de la galaxia; (3) tipo de galaxia según RC3 (Tercera Referencia del Catálogo de Galaxias Brillantes); (4) corrimiento al rojo (z) en base a una constante de Hubble $\mathrm{H}_{0}=70 \mathrm{~km} \mathrm{~s}^{-1}$ $\mathrm{Mpc}^{-1}$; (5) distancia a la galaxia, en Mega pársecs; (6) tipo del núcleo, según el artículo de Ho, Phillipenko \& Sargent de 1997; (7) masa calculada del agujero negro súper masivo, en masas solares; (8) cota mínima de error para $\mathrm{M}_{\mathrm{BH}}$, en masas solares; (9) cota máxima de error para $\mathrm{M}_{\mathrm{BH}}$, en masas solares; (10) método empleado para el

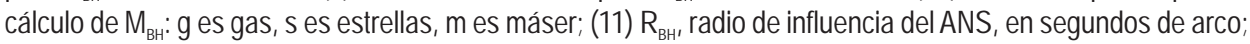
(12) Nres $=2 R_{\text {вн }}$ / Rres, donde Rres es la resolución espacial de las observaciones (la medición se considera segura si Nres $>1$ ).

Las referencias corresponden al siguiente código de colores, para las 3 tablas:

\begin{tabular}{|cc|l|l|l|}
\hline NED & HFS97 & Ferrarese \& Merrit 2000 & Haring \& Rix 2004 & Wu\& Ca0 2005 \\
\hline Satyapal + 2004 & Gebhardt +2000 & Marconi \& Hunt 2003 & Dudik+ 2005 & Gallo + 2008 \\
\hline
\end{tabular}




\begin{tabular}{|c|c|c|c|c|c|c|c|c|c|c|c|}
\hline \multicolumn{12}{|c|}{ TABLA 1: GALAXIAS ELIPTICAS (columnas 1 a 12) } \\
\hline No. & Nombre & $\begin{array}{l}\text { Tipo } \\
\text { Hubble }\end{array}$ & $\begin{array}{c}Z \\
\text { Estimado } \\
\text { con } \\
\text { Ho=70 } \\
\mathrm{Km} / \mathrm{s} / \mathrm{mpc}\end{array}$ & $\begin{array}{c}\mathrm{D}, \mathrm{Mpc} \\
(\mathrm{HO}=70)\end{array}$ & $\begin{array}{c}\text { Tipo } \\
\text { nuclear }\end{array}$ & $\begin{array}{l}\mathrm{M}_{\mathrm{BH}}, \\
\text { Msol }\end{array}$ & $\begin{array}{l}\text { Error } \\
\mathrm{M}_{\mathrm{BH}},-\end{array}$ & $\begin{array}{l}\text { Error } \\
\mathrm{M}_{\mathrm{BH}},+\end{array}$ & Ref & $\mid \begin{array}{c}\mathrm{R}_{\mathrm{BH}}, \\
\operatorname{arcsec}\end{array}$ & Nres \\
\hline (1) & (2) & (3) & $(4)$ & (5) & (6) & (7) & (8) & (9) & (10) & (11) & (12) \\
\hline 14 & 3608 & E2 & 0.005 & 22.9 & & $\begin{array}{l}1.90 \mathrm{E} \\
+08 \\
\end{array}$ & $\begin{array}{l}6.00 \mathrm{E} \\
+07 \\
\end{array}$ & $\begin{array}{l}1.00 \mathrm{E} \\
+08\end{array}$ & $\mathrm{~S}$ & 0.02 & 4.4 \\
\hline 15 & 4125 & E6;pcc & 0.005 & 19.3929 & & $\begin{array}{l}3.16 \mathrm{E} \\
+08\end{array}$ & & & & & \\
\hline 16 & 4261 & E2 & 0.007 & 31.6 & & $\begin{array}{l}5.20 \mathrm{E} \\
+08\end{array}$ & $\begin{array}{l}1.10 \mathrm{E} \\
+08\end{array}$ & $\begin{array}{l}1.00 \mathrm{E} \\
+08\end{array}$ & G & 0.15 & 2.9 \\
\hline 17 & 4278 & E1-2 & 0.004 & 17.25 & & $\begin{array}{l}1.58 \mathrm{E} \\
+09 \\
\end{array}$ & & & & & \\
\hline 18 & 4291 & E2 & 0.006 & 26.2 & & $\begin{array}{l}3.10 \mathrm{E} \\
+08 \\
\end{array}$ & $\begin{array}{l}2.30 \mathrm{E} \\
+08\end{array}$ & $\begin{array}{l}8.00 \mathrm{E} \\
+07\end{array}$ & $\mathrm{~S}$ & 0.18 & 3.6 \\
\hline 19 & 4374 & E1c & 0.004 & 18.9671 & & $\begin{array}{l}1.70 \mathrm{E} \\
+09\end{array}$ & $\begin{array}{l}\begin{array}{l}6.70 E \\
+08\end{array} \\
\end{array}$ & $\begin{array}{l}1.20 \mathrm{E} \\
+09\end{array}$ & G & & \\
\hline 20 & 4473 & E5 & 0.004 & 15.7 & & $\begin{array}{l}1.10 \mathrm{E} \\
+08 \\
\end{array}$ & \begin{tabular}{|l|}
$8.00 \mathrm{E}$ \\
+07 \\
\end{tabular} & \begin{tabular}{|l|}
$4.00 \mathrm{E}$ \\
+07 \\
\end{tabular} & $\mathrm{~S}$ & 0.17 & 3.4 \\
\hline 21 & 4486 & E0pec & 0.004 & 16.9386 & & $\begin{array}{l}3.57 \mathrm{E} \\
+09 \\
\end{array}$ & $\begin{array}{l}1.02 \mathrm{E} \\
+09 \\
\end{array}$ & \begin{tabular}{|l|}
$1.02 \mathrm{E}$ \\
+09 \\
\end{tabular} & G & & \\
\hline 22 & 4564 & E3 & 0.004 & 15 & & $\begin{array}{l}5.60 \mathrm{E} \\
+07\end{array}$ & $\begin{array}{l}8.00 E \\
+06\end{array}$ & $\begin{array}{l}\begin{array}{l}3.00 E \\
+06\end{array} \\
\end{array}$ & $\mathrm{~S}$ & 0.13 & 2.5 \\
\hline 23 & 4649 & E1 & 0.004 & 16.8 & & $\begin{array}{l}2.00 \mathrm{E} \\
+09 \\
\end{array}$ & $\begin{array}{l}6.00 \mathrm{E} \\
+08 \\
\end{array}$ & \begin{tabular}{|l|}
$4.00 \mathrm{E}$ \\
+08 \\
\end{tabular} & $\mathrm{~S}$ & 0.71 & 14 \\
\hline 24 & 4696 & $E+1 ; p$. & 0.010 & 42.3214 & & $\begin{array}{l}\begin{array}{l}3.98 \mathrm{E} \\
+08\end{array} \\
\end{array}$ & & & & & \\
\hline 25 & 4697 & E4 & 0.003 & 11.7 & & $\begin{array}{l}1.70 \mathrm{E} \\
+08 \\
\end{array}$ & \begin{tabular}{|l|}
$1.00 \mathrm{E}$ \\
+07 \\
\end{tabular} & \begin{tabular}{|l|}
$\begin{array}{l}2.00 \mathrm{E} \\
+07\end{array}$ \\
\end{tabular} & $\mathrm{~S}$ & 0.41 & 8.2 \\
\hline 26 & 4742 & E4 & 0.004 & 15.5 & & $\begin{array}{l}1.40 \mathrm{E} \\
+07\end{array}$ & \begin{tabular}{|l|}
$5.00 \mathrm{E}$ \\
+06 \\
\end{tabular} & \begin{tabular}{|l|}
$4.00 \mathrm{E}$ \\
+06
\end{tabular} & $\mathrm{~S}$ & 0.1 & 2 \\
\hline 27 & 5845 & E3 & 0.006 & 25.9 & & $\begin{array}{l}2.40 \mathrm{E} \\
+08 \\
\end{array}$ & \begin{tabular}{|l|}
$1.40 \mathrm{E}$ \\
+08 \\
\end{tabular} & \begin{tabular}{|l|}
$\begin{array}{l}4.00 \mathrm{E} \\
+07\end{array}$ \\
\end{tabular} & $\mathrm{~S}$ & 0.15 & 1.4 \\
\hline 28 & 6251 & E2 & 0.025 & 107 & & $\begin{array}{l}\begin{array}{l}6.10 \mathrm{E} \\
+08\end{array} \\
\end{array}$ & $\begin{array}{l}2.10 \mathrm{E} \\
+08 \\
\end{array}$ & \begin{tabular}{|l|}
$2.00 \mathrm{E}$ \\
+08
\end{tabular} & $\mathrm{G}$ & 0.06 & 1.2 \\
\hline 29 & 7052 & E4 & 0.017 & 71.4 & & $\begin{array}{l}4.00 \mathrm{E} \\
+08 \\
\end{array}$ & $\begin{array}{l}1.60 \mathrm{E} \\
+08 \\
\end{array}$ & \begin{tabular}{|l|}
$2.80 \mathrm{E}$ \\
+08 \\
\end{tabular} & $\bar{G}$ & 0.07 & 0.5 \\
\hline 30 & $1154 a$ & & 0.004 & 17.2179 & & $\begin{array}{l}7.94 \mathrm{E} \\
+07 \\
\end{array}$ & & & & & \\
\hline 31 & $1226 a$ & & 0.004 & 18.3643 & & \begin{tabular}{|l|}
$1.26 \mathrm{E}$ \\
+09 \\
\end{tabular} & & & & & \\
\hline 32 & $1231 a$ & & 0.004 & 16.3607 & & $\begin{array}{l}1.26 \mathrm{E} \\
+08\end{array}$ & & & & & \\
\hline
\end{tabular}




\begin{tabular}{|c|c|c|c|c|c|c|c|c|c|c|c|}
\hline \multicolumn{12}{|c|}{ TABLA 1: GALAXIAS ELIPTICAS (columnas 1 a 12) } \\
\hline No. & Nombre & $\begin{array}{l}\text { Tipo } \\
\text { Hubble }\end{array}$ & $\begin{array}{c}Z \\
\text { Estimado } \\
\text { con } \\
\mathrm{Ho}=70 \\
\mathrm{Km} / \mathrm{s} / \mathrm{mpc}\end{array}$ & $\begin{array}{r}\mathrm{D}, \mathrm{Mpc} \\
(\mathrm{H} 0=70) \\
\end{array}$ & $\begin{array}{c}\text { Tipo } \\
\text { nuclear }\end{array}$ & $\begin{array}{l}M_{\text {BH, }} \\
\text { Msol }\end{array}$ & $\begin{array}{l}\text { Error } \\
\mathrm{M}_{\mathrm{BH}} \text { - }^{-}\end{array}$ & $\begin{array}{l}\text { Error } \\
\mathrm{M}_{\mathrm{BH}}+\end{array}$ & Ref & $\begin{array}{c}\mathrm{R}_{\mathrm{BH}} \\
\operatorname{arcsec}\end{array}$ & Nres \\
\hline (1) & $(2)$ & (3) & $(4)$ & $(5)$ & (6) & (7) & $(8)$ & (9) & $(10)$ & (11) & $(12)$ \\
\hline 33 & $1297 a$ & & 0.004 & 17.4536 & & $\begin{array}{l}6.31 \mathrm{E} \\
+07\end{array}$ & & & & & \\
\hline 34 & $1316 \mathrm{a}$ & & 0.004 & 18.45 & & $\begin{array}{l}2.51 \mathrm{E} \\
+09\end{array}$ & & & & & \\
\hline 35 & $1535 a$ & & 0.004 & 17.6786 & & $\begin{array}{l}1.58 \mathrm{E} \\
+09 \\
\end{array}$ & & & & & \\
\hline 36 & $1632 \mathrm{a}$ & & 0.004 & 16.9821 & & \begin{tabular}{|l|}
$5.01 \mathrm{E}$ \\
+08 \\
\end{tabular} & & & & & \\
\hline 37 & $1664 a$ & & 0.004 & 16.9821 & & \begin{tabular}{|l|}
$5.01 \mathrm{E}$ \\
+07 \\
\end{tabular} & & & & & \\
\hline 38 & $1903 a$ & & 0.004 & 15.9964 & & \begin{tabular}{|l|}
$3.16 \mathrm{E}$ \\
+08 \\
\end{tabular} & & & & & \\
\hline 39 & $1978 a$ & & 0.004 & 18.5357 & & \begin{tabular}{|l|}
$2.51 \mathrm{E}$ \\
+09
\end{tabular} & & & & & \\
\hline 40 & $2095 a$ & & 0.004 & 17.6786 & & $\begin{array}{l}3.98 \mathrm{E} \\
+07 \\
\end{array}$ & & & & & \\
\hline 41 & $685 a$ & & 0.004 & 17.6786 & & \begin{tabular}{|l|}
$1.58 \mathrm{E}$ \\
+08
\end{tabular} & & & & & \\
\hline 42 & $731 a$ & & 0.006 & 24.9964 & & $\begin{array}{l}6.31 \mathrm{E} \\
+08\end{array}$ & & & & & \\
\hline 43 & $763 a$ & & 0.005 & 19.7679 & & $\begin{array}{l}1.00 \mathrm{E} \\
+09\end{array}$ & & & & & \\
\hline 44 & $798 a$ & & 0.004 & 19.1357 & & \begin{tabular}{|l|}
$2.00 \mathrm{E}$ \\
+08
\end{tabular} & & & & & \\
\hline 45 & $881 a$ & & 0.004 & 18.0321 & & $\begin{array}{l}3.98 \mathrm{E} \\
+08\end{array}$ & & & & & \\
\hline 46 & $\begin{array}{c}\text { Cygnus } \\
\text { A }\end{array}$ & $E$ & 0.056 & 240 & & $\begin{array}{l}2.90 \mathrm{E} \\
+09 \\
\end{array}$ & \begin{tabular}{|l|}
$.00 \mathrm{E}$ \\
+08 \\
\end{tabular} & \begin{tabular}{|l|}
$\begin{array}{l}7.00 \mathrm{E} \\
+08\end{array}$ \\
\end{tabular} & G & 0.15 & 2.9 \\
\hline 47 & IC1459 & E3 & 0.007 & 29.2 & & \begin{tabular}{|l|}
$1.50 \mathrm{E}$ \\
+09
\end{tabular} & \begin{tabular}{|l|l|}
$\begin{array}{l}1.00 \mathrm{E} \\
+09\end{array}$ \\
+09
\end{tabular} & \begin{tabular}{|l|}
$1.00 \mathrm{E}$ \\
+09
\end{tabular} & $S$ & 0.39 & 7.8 \\
\hline 48 & M32 & $\bar{E} 2 p$ & 0.00021 & 0.91429 & & $\begin{array}{l}3.70 \mathrm{E} \\
+06 \\
\end{array}$ & \begin{tabular}{|l|}
$2.40 \mathrm{E}$ \\
+06 \\
\end{tabular} & \begin{tabular}{|l|}
$4.40 \mathrm{E}$ \\
+06 \\
\end{tabular} & $S$ & & \\
\hline 49 & $\begin{array}{c}\text { M84 } \\
(4374)\end{array}$ & E1 & 0.004 & 18.4 & & $\begin{array}{l}1.00 \mathrm{E} \\
+09 \\
\end{array}$ & \begin{tabular}{|l|}
$6.00 \mathrm{E}$ \\
+08 \\
\end{tabular} & \begin{tabular}{|l|}
$2.00 \mathrm{E}$ \\
+09 \\
\end{tabular} & G & 0.55 & 11 \\
\hline 50 & $\begin{array}{c}\text { M87 } \\
(4486)\end{array}$ & E0 & 0.004 & 16.1 & & $\begin{array}{l}3.40 \mathrm{E} \\
+09\end{array}$ & \begin{tabular}{|l|}
$1.00 \mathrm{E}$ \\
+06 \\
+06
\end{tabular} & \begin{tabular}{|l|}
$1.00 \mathrm{E}$ \\
+06
\end{tabular} & G & 1.33 & 33 \\
\hline
\end{tabular}




\begin{tabular}{|c|c|c|c|c|c|c|c|c|c|c|}
\hline \multicolumn{7}{|c|}{ TABLA 2: GALAXIAS ELIPTICAS (columnas 13 a 22) } \\
\hline No. & $\sigma_{c}, \mathrm{~km} / \mathrm{s}$ & $\begin{array}{c}\text { Error en } \\
\sigma_{\mathrm{c}}( \pm)\end{array}$ & Re, kpc & $\begin{array}{c}\text { Error Re } \\
( \pm)\end{array}$ & $\begin{array}{c}\text { Lfir, Lsol } \\
\text { L bulbo }\end{array}$ & $\begin{array}{c}\text { Mbul, } \\
\text { Lsol } \\
10^{\circ} \\
\text { Msol }\end{array}$ & $\begin{array}{c}\text { Error } \\
\text { Mbul, } \\
10^{\circ}( \pm)\end{array}$ & $\begin{array}{c}\text { Gamma } \\
=\text { M/L } \\
\text { (Msol/ } \\
\text { Lsol) }\end{array}$ & $\begin{array}{c}\text { Banda } \\
\text { en que } \\
\text { se } \\
\text { mide } \\
\text { gamma }\end{array}$ \\
\hline$(1)$ & $(13)$ & $(14)$ & $(15)$ & $(16)$ & $(17)$ & $(18)$ & $(19)$ & $(20)$ & $(21)$ & $(22)$ \\
\hline 1 & 76 & & & & & & & & & \\
\hline 2 & 209 & & 20 & 5 & & $\begin{array}{l}2.90 \mathrm{E} \\
+10\end{array}$ & 620 & 170 & 4.5 & $\mathrm{R}$ \\
\hline 3 & & & & & $\begin{array}{l}1.26 \mathrm{E}+ \\
09\end{array}$ & & & & & \\
\hline 4 & 121 & 25 & & & & & & & & \\
\hline 5 & 180 & 18 & & & & & & & & \\
\hline 6 & 153 & 15 & & & & & & & & \\
\hline 7 & 104 & 11 & & & & & & & & \\
\hline 8 & 89 & 10 & & & & & & & & \\
\hline 9 & 79 & 5 & & & & & & & & \\
\hline 10 & 200 & 10 & & & & & & & & \\
\hline 11 & 175 & & 3 & 0.8 & & $\begin{array}{l}1.20 \mathrm{E} \\
+10\end{array}$ & 65 & & 6.6 & $\mathrm{~V}$ \\
\hline 12 & 145 & & 5.4 & 1.3 & & $\begin{array}{l}6.40 \mathrm{E} \\
+09\end{array}$ & 78 & 17 & 2.5 & $\mathrm{~V}$ \\
\hline 13 & 206 & & 2.9 & 0.7 & & $\begin{array}{l}1.70 \mathrm{E} \\
+10\end{array}$ & 85 & 21 & 4 & $\mathrm{R}$ \\
\hline 14 & 182 & & 4.3 & 1.1 & & $\begin{array}{l}1.90 \mathrm{E} \\
+10\end{array}$ & 99 & 23 & 5.2 & $\mathrm{~V}$ \\
\hline
\end{tabular}

${ }^{9}$ (13) dispersión central de velocidades, en km/s; (14) cota de error en la medición de sigma, en km/s; (15) Re, radio efectivo del bulbo, en kpc; (16) cotas mínima y máxima de error en el cálculo de Re; (17) luminosidad en lejano infrarrojo, en luminosidades solares; (18) luminosidad del bulbo, en luminosidades solares; (19) Mbul, masa del bulbo, en miles de millones de masas solares; (20) cotas de error en el cálculo de Mbul; (21) Gamma, relación masa-luminosidad de toda la galaxia, en masas solares sobre luminosidades solares; (22) banda en la que se mide el valor de gamma: V es luz visible, R es rojo, I es infrarrojo.

Las referencias corresponden al siguiente código de colores, para las 3 tablas:

\begin{tabular}{|cc|l|l|l|}
\hline NED & HFS97 & Ferrarese \& Merrit 2000 & Haring \& Rix 2004 & Wu\& Ca0 2005 \\
\hline Satyapal + 2004 & Gebhardt +2000 & Marconi \& Hunt 2003 & Dudik+ 2005 & Gallo + 2008 \\
\hline
\end{tabular}




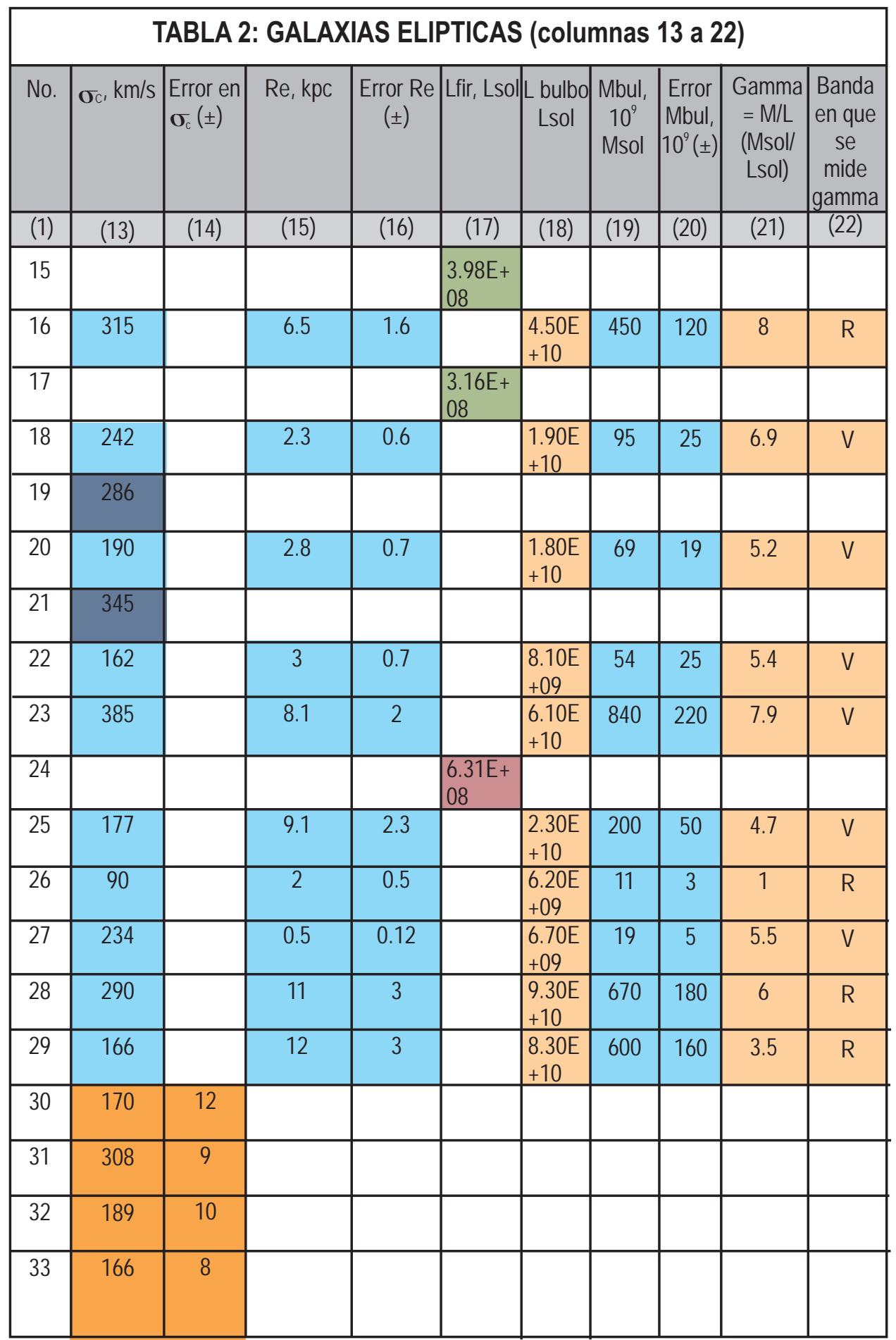




\begin{tabular}{|c|c|c|c|c|c|c|c|c|c|c|}
\hline \multicolumn{11}{|c|}{ TABLA 2: GALAXIAS ELIPTICAS (columnas 13 a 22) } \\
\hline No. & $\sigma_{c}, \mathrm{~km} / \mathrm{s}$ & $\begin{array}{l}\text { Error en } \\
\sigma_{\mathrm{c}}( \pm)\end{array}$ & $\mathrm{Re}, \mathrm{kpc}$ & $\begin{array}{c}\text { Error Re } \\
\quad( \pm)\end{array}$ & Lfir, Lsol & $\begin{array}{c}\text { L bulbo } \\
\text { Lsol }\end{array}$ & $\begin{array}{c}\text { Mbul, } \\
10^{\circ} \\
\text { Msol }\end{array}$ & $\begin{array}{c}\text { Error } \\
\text { Mbul, } \\
10^{\circ}( \pm)\end{array}$ & $\begin{array}{c}\text { Gamma } \\
=\mathrm{M} / \mathrm{L} \\
\text { (Msol/ } \\
\text { Lsol) }\end{array}$ & $\begin{array}{c}\text { Banda } \\
\text { en que } \\
\text { se } \\
\text { mide } \\
\text { gamma }\end{array}$ \\
\hline (1) & (13) & (14) & (15) & (16) & (17) & (18) & (19) & (20) & $\begin{array}{l}(21) \\
\end{array}$ & $(22)$ \\
\hline 34 & 355 & 8 & & & & & & & & \\
\hline 35 & 316 & 7 & & & & & & & & \\
\hline 36 & 257 & 18 & & & & & & & & \\
\hline 37 & 157 & 9 & & & & & & & & \\
\hline 38 & 233 & 7 & & & & & & & & \\
\hline 39 & 347 & 9 & & & & & & & & \\
\hline 40 & 147 & 10 & & & & & & & & \\
\hline 41 & 198 & 9 & & & & & & & & \\
\hline 42 & 261 & 7 & & & & & & & & \\
\hline 43 & 297 & 7 & & & & & & & & \\
\hline 44 & 205 & 8 & & & & & & & & \\
\hline 45 & 245 & 11 & & & & & & & & \\
\hline 46 & 270 & & 31 & 8 & & & 1600 & 1100 & & \\
\hline 47 & 340 & & 8.2 & 2 & $\begin{array}{l}3.98 \mathrm{E}+ \\
08\end{array}$ & \begin{tabular}{|l|}
$6.90 \mathrm{E}$ \\
+10
\end{tabular} & 660 & 180 & 4.2 & $\mathrm{R}$ \\
\hline 48 & & & & & & & & & & \\
\hline 49 & 296 & & 8.2 & 2.1 & & & 500 & 140 & & \\
\hline 50 & 375 & & 6.4 & 1.6 & & $\begin{array}{l}2.00 \mathrm{E} \\
+11\end{array}$ & 620 & 170 & 3 & I \\
\hline
\end{tabular}




\begin{tabular}{|c|c|c|c|c|c|c|c|c|c|c|c|}
\hline \multicolumn{12}{|c|}{ TABLA 2: GALAXIAS ESPIRALES (columnas 1 a 12) } \\
\hline No. & Nombre & \begin{tabular}{|l} 
Tipo \\
Hubble
\end{tabular} & $\begin{array}{c}\mathrm{Z} \\
\text { Estimado } \\
\text { con } \mathrm{Ho}=70 \\
\mathrm{Km} / \mathrm{s} / \mathrm{mpc}\end{array}$ & $\begin{array}{l}\mathrm{D}, \mathrm{Mpc} \\
(\mathrm{HO}=70)\end{array}$ & $\begin{array}{c}\text { Tipo } \\
\text { nuclear }\end{array}$ & $\begin{array}{l}M_{\text {BH, }} \\
\text { Msol }\end{array}$ & $\begin{array}{l}\text { Error } \\
\mathrm{M}_{\mathrm{BH}} \text {, - }\end{array}$ & $\begin{array}{c}\text { Error } \\
\mathrm{M}_{\mathrm{BH}}+\end{array}$ & Ref & $\begin{array}{c}\mathrm{R}_{\mathrm{Bн}}, \\
\operatorname{arcsec}\end{array}$ & Nres \\
\hline$(1)$ & $(2)$ & (3) & $(4)$ & $(5)$ & $(6)$ & $(7)$ & $(8)$ & $(9)$ & $(10)$ & $(11)$ & $(12)$ \\
\hline 51 & 253 & $\mathrm{SAB}(\mathrm{S}) \mathrm{C}$ & 0.001 & 2.8 & & $\begin{array}{l}1.17 \mathrm{E} \\
+07\end{array}$ & & & & & \\
\hline 52 & 404 & $\mathrm{SA}(\mathrm{s}) 0$ & 0.001 & 2.6 & & $\begin{array}{l}\begin{array}{l}7.59 \mathrm{E} \\
+05\end{array} \\
+05\end{array}$ & & & & & \\
\hline 53 & 660 & $\begin{array}{l}\mathrm{SB}(\mathrm{s}) \mathrm{a} ; \\
\text { p. }\end{array}$ & 0.003 & 12.1 & & $\begin{array}{l}2.24 \mathrm{E} \\
+07\end{array}$ & & & & & \\
\hline 54 & 835 & $\begin{array}{c}S A B(r) a \\
b ; p .\end{array}$ & 0.014 & 58.2 & & $\begin{array}{l}9.33 \mathrm{E} \\
+08 \\
\end{array}$ & & & & & \\
\hline 55 & 838 & $\begin{array}{c}\text { SAB(r)a } \\
\text { b;pcc }\end{array}$ & 0.014 & 58.2 & & & & & & & \\
\hline 56 & 1023 & Sb0 & 0.003 & 11.4 & A & $\begin{array}{l}4.40 \mathrm{E} \\
+07\end{array}$ & \begin{tabular}{|l|}
$5.00 \mathrm{E}$ \\
+06
\end{tabular} & \begin{tabular}{|l|}
$5.00 \mathrm{E}$ \\
+06
\end{tabular} & $\mathrm{~S}$ & 0.08 & 1.6 \\
\hline 57 & 1055 & $\mathrm{SBb} ; \mathrm{sp}$ & 0.003 & 14.3 & & $\begin{array}{l}3.39 \mathrm{E} \\
+06\end{array}$ & & & & & \\
\hline 58 & 1365 & $\begin{array}{c}\left(\mathrm{R}^{\prime}\right) \mathrm{SBb}( \\
\mathrm{s}) \mathrm{b}\end{array}$ & 0.005 & 22.2 & Sy1.8 & & & & & & \\
\hline 59 & 2787 & Sb0 & 0.002 & 7.5 & L1.9 & $\begin{array}{l}4.10 \mathrm{E} \\
+07\end{array}$ & \begin{tabular}{|l|}
$5.00 \mathrm{E}$ \\
+06
\end{tabular} & \begin{tabular}{|l|}
$4.00 \mathrm{E}$ \\
+06
\end{tabular} & $G$ & 0.25 & 5 \\
\hline 60 & 3031 & $\mathrm{SA}(\mathrm{S}) \mathrm{ab}$ & 0.001 & 3.9 & S1.5 & $\begin{array}{l}7.60 \mathrm{E} \\
+07\end{array}$ & \begin{tabular}{|l|}
$\begin{array}{l}1.10 \mathrm{E} \\
+06\end{array}$ \\
\end{tabular} & \begin{tabular}{|l|}
$2.20 \mathrm{E}$ \\
+06
\end{tabular} & & 0.63 & 13 \\
\hline 61 & 3079 & $\mathrm{SB}(\mathrm{s}) \mathrm{C}$ & 0.004 & 16.1 & & \begin{tabular}{|l|}
$4.47 \mathrm{E}$ \\
+07
\end{tabular} & & & & & \\
\hline 62 & 3115 & so & 0.002 & 9.7 & $\bar{A}$ & $\begin{array}{l}9.10 \mathrm{E} \\
+08\end{array}$ & $\begin{array}{l}\begin{array}{l}2.80 \mathrm{E} \\
+08\end{array} \\
+\end{array}$ & \begin{tabular}{|l|}
$9.90 \mathrm{E}$ \\
+08
\end{tabular} & $\mathrm{~S}$ & 1.57 & 15 \\
\hline 63 & 3125 & $\mathrm{~S}$ & 0.003 & 12.3 & & & & & & & \\
\hline 64 & 3245 & So & 0.005 & 20.9 & T2 & $\begin{array}{l}2.10 \mathrm{E} \\
+08\end{array}$ & $\begin{array}{l}5.00 \mathrm{E} \\
+07\end{array}$ & $\begin{array}{l}5.00 \mathrm{E} \\
+07\end{array}$ & G & 0.21 & 4.2 \\
\hline
\end{tabular}

${ }^{10}$ (1) número asignado; (2) nombre más común de la galaxia; (3) tipo de galaxia según la clasificación de Hubble; (4) corrimiento al rojo (z) en base a una constante de Hubble $\mathrm{H}_{0}=70 \mathrm{~km} \mathrm{~s}^{-1} \mathrm{Mpc}^{-1}$; (5) distancia a la galaxia, en Mega pársecs; (6) tipo del núcleo, según el artículo de Ho, Phillipenko \& Sargent de 1997; (7) masa calculada del agujero negro súper masivo, en masas solares; (8) cota mínima de error para $\mathrm{M}_{\mathrm{BH}}$, en masas solares; (9) cota máxima de error para $\mathrm{M}_{\mathrm{BH}}$, en masas solares; (10) método empleado para el cálculo de $\mathrm{M}_{\mathrm{Bн}}$ : $g$ es gas, $\mathrm{s}$ es estrellas, m es máser; (11) $R_{B H}$, radio de influencia del ANS, en segundos de $\operatorname{arco}$; (12) Nres $=2 R_{B H} /$ Rres, donde Rres es la resolución espacial de las observaciones (la medición se considera segura si Nres >1).

Las referencias corresponden al siguiente código de colores, para las 3 tablas:

\begin{tabular}{|cc|l|l|l|}
\hline NED & HFS97 & Ferrarese \& Merrit 2000 & Haring \& Rix 2004 & Wu\& Cao 2005 \\
\hline Satyapal + 2004 & Gebhardt +2000 & Marconi \& Hunt 2003 & Dudik+ 2005 & Gallo + 2008 \\
\hline
\end{tabular}




\begin{tabular}{|c|c|c|c|c|c|c|c|c|c|c|c|}
\hline \multicolumn{12}{|c|}{ TABLA 2: GALAXIAS ESPIRALES (columnas 1 a 12) } \\
\hline No. & Nombre & $\begin{array}{c}\text { Tipo } \\
\text { Hubble }\end{array}$ & $\begin{array}{c}\mathrm{Z} \\
\text { Estimado } \\
\text { con Ho=70 } \\
\mathrm{Km} / \mathrm{s} / \mathrm{mpc}\end{array}$ & $\begin{array}{c}\mathrm{D}, \mathrm{Mpc} \\
(\mathrm{H} 0=70)\end{array}$ & $\begin{array}{c}\text { Tipo } \\
\text { nuclear }\end{array}$ & $\begin{array}{l}\mathrm{M}_{\mathrm{BH}}, \\
\text { Msol }\end{array}$ & $\begin{array}{l}\text { Error } \\
\mathrm{M}_{\mathrm{BH}},-\end{array}$ & $\begin{array}{l}\text { Error } \\
\mathrm{M}_{\mathrm{BH}},+\end{array}$ & Ref & $\begin{array}{c}\mathrm{R}_{\mathrm{BH}}, \\
\operatorname{arcsec}\end{array}$ & Nres \\
\hline (1) & (2) & (3) & \begin{tabular}{|l|}
$(4)$ \\
\end{tabular} & (5) & (6) & (7) & (8) & (9) & (10) & (11) & (12) \\
\hline 65 & 3384 & SO & 0.003 & 11.6 & $A$ & $\begin{array}{l}1.60 \mathrm{E} \\
+07\end{array}$ & $\begin{array}{l}2.00 \mathrm{E} \\
+06\end{array}$ & $\begin{array}{l}\begin{array}{l}1.00 E \\
+06\end{array} \\
\end{array}$ & $\mathrm{~S}$ & 0.06 & 1.2 \\
\hline 66 & 4013 & $\mathrm{Sb}$ & 0.003 & 11.9 & & & & & & & \\
\hline 67 & 4102 & $\begin{array}{c}\mathrm{SAB}(\mathrm{s}) \mathrm{b} \\
?\end{array}$ & 0.003 & 12.0 & & & & & & & \\
\hline 68 & 4258 & Sbc & 0.002 & 7.2 & S1.9 & $\begin{array}{l}3.90 \mathrm{E} \\
+07\end{array}$ & $\begin{array}{l}1.00 \mathrm{E} \\
+06\end{array}$ & $\begin{array}{l}1.00 E \\
+06\end{array}$ & $M$ & 0.28 & 71 \\
\hline 69 & 4314 & $\mathrm{SB}(\mathrm{rs}) \mathrm{a}$ & 0.003 & 13.7 & & $\begin{array}{l}1.66 \mathrm{E} \\
+07\end{array}$ & & & & & \\
\hline 70 & 4342 & SO & 0.003 & 11.4 & & $\begin{array}{l}2.20 \mathrm{E} \\
+08\end{array}$ & $\begin{array}{l}8.00 \mathrm{E} \\
+07\end{array}$ & $\begin{array}{l}1.30 \mathrm{E} \\
+08\end{array}$ & $S$ & 0.34 & 0.8 \\
\hline 71 & 4350 & $\mathrm{SAO}$ & 0.004 & 17.8 & & $\begin{array}{l}9.77 \mathrm{E} \\
+07\end{array}$ & & & & & \\
\hline 72 & 4419 & $\mathrm{SB}(\mathrm{s}) \mathrm{a}$ & 0.004 & 18.0 & & $\begin{array}{l}8.71 \mathrm{E} \\
+06\end{array}$ & & & & & \\
\hline 73 & 4459 & SO & 0.004 & 16.1 & T2: & $\begin{array}{l}7.00 \mathrm{E} \\
+07\end{array}$ & $\begin{array}{l}1.30 \mathrm{E} \\
+07\end{array}$ & $\begin{array}{l}1.30 \mathrm{E} \\
+07\end{array}$ & $\bar{G}$ & 0.11 & 2.2 \\
\hline 74 & 4527 & $\begin{array}{c}\mathrm{SAB}(\mathrm{s}) \mathrm{b} \\
\mathrm{c}\end{array}$ & 0.006 & 24.9 & & $\begin{array}{l}1.78 \mathrm{E} \\
+08\end{array}$ & & & & & \\
\hline 75 & 4594 & $\mathrm{Sa}$ & 0.002 & 9.8 & L2 & $\begin{array}{l}1.00 \mathrm{E} \\
+09\end{array}$ & $\begin{array}{l}7.00 \mathrm{E} \\
+08\end{array}$ & $\begin{array}{l}1.00 \mathrm{E} \\
+09\end{array}$ & $S$ & 1.57 & 5 \\
\hline 76 & 4596 & SBO & 0.007 & 27.9 & L2:: & $\begin{array}{l}7.80 \mathrm{E} \\
+07\end{array}$ & $\begin{array}{l}\begin{array}{l}3.30 \mathrm{E} \\
+07\end{array} \\
\end{array}$ & $\begin{array}{l}4.20 \mathrm{E} \\
+07\end{array}$ & $\mathrm{G}$ & 0.11 & 2.1 \\
\hline 77 & 4651 & $\mathrm{SA}(\mathrm{rs}) \mathrm{C}$ & 0.003 & 11.5 & & & & & & & \\
\hline 78 & 4666 & $\mathrm{SABC}$ & 0.005 & 21.8 & & & & & & & \\
\hline 79 & 4713 & $\begin{array}{c}\mathrm{SAB}(\mathrm{rs}) \\
\mathrm{d}\end{array}$ & 0.002 & 9.3 & & & & & & & \\
\hline 80 & 5005 & $\begin{array}{c}\mathrm{SAB}(\mathrm{rs}) \\
\mathrm{bc}\end{array}$ & 0.003 & 13.5 & & & & & & & \\
\hline 81 & $\begin{array}{l}\text { Cen A } \\
\text { (5128) }\end{array}$ & S0 & 0.001 & 4.2 & & $\begin{array}{l}2.40 \mathrm{E} \\
+08\end{array}$ & $\begin{array}{l}1.70 \mathrm{E} \\
+08\end{array}$ & $\begin{array}{l}3.60 \mathrm{E} \\
+08\end{array}$ & $\mathrm{~S}$ & 2.25 & 9 \\
\hline 82 & 5252 & S0 & 0.023 & 96.8 & & $\begin{array}{l}1.00 \mathrm{E} \\
+09 \\
\end{array}$ & $\begin{array}{l}4.00 \mathrm{E} \\
+08 \\
\end{array}$ & $\begin{array}{l}2.00 \mathrm{E} \\
+08 \\
\end{array}$ & $\bar{G}$ & 0.25 & 5.1 \\
\hline 83 & 5678 & $\begin{array}{c}S A B(r s) \\
b\end{array}$ & 0.006 & 27.4 & & & & & & & \\
\hline 84 & 5899 & $\begin{array}{c}\mathrm{SAB}(\mathrm{rs}) \\
\mathrm{c}\end{array}$ & 0.009 & 36.6 & & & & & & & \\
\hline
\end{tabular}




\begin{tabular}{|c|c|c|c|c|c|c|c|c|c|c|c|}
\hline \multicolumn{12}{|c|}{ TABLA 2: GALAXIAS ESPIRALES (columnas 1 a 12) } \\
\hline No. & Nombre & $\begin{array}{c}\text { Tipo } \\
\text { Hubble }\end{array}$ & $\begin{array}{c}\mathrm{Z} \\
\text { Estimado } \\
\text { con } \mathrm{Ho}=70 \\
\mathrm{Km} / \mathrm{s} / \mathrm{mpc}\end{array}$ & $\begin{array}{l}\mathrm{D}, \mathrm{Mpc} \\
(\mathrm{H} 0=70) \\
\end{array}$ & $\begin{array}{c}\text { Tipo } \\
\text { nuclear }\end{array}$ & $\begin{array}{l}\mathrm{M}_{\mathrm{BH}}, \\
\mathrm{Msol}\end{array}$ & $\begin{array}{l}\text { Error } \\
\mathrm{M}_{\mathrm{BH}} \text {, }^{-}\end{array}$ & $\begin{array}{l}\text { Error } \\
\mathrm{M}_{\mathrm{BH}}+\end{array}$ & Ref & $\left|\begin{array}{c}\mathrm{R}_{\text {вH }} \\
\operatorname{arcsec}\end{array}\right|$ & Nres \\
\hline (1) & $(2)$ & $(3)$ & $(4)$ & $(5)$ & (6) & $(7)$ & (8) & (9) & $(10)$ & (11) & $(12)$ \\
\hline 85 & 5954 & SAa:;p. & 0.007 & 28.1 & & $\begin{array}{l}6.46 \mathrm{E} \\
+06\end{array}$ & & & & & \\
\hline 86 & 6500 & SAab & 0.010 & 43.0 & & $\begin{array}{l}6.61 \mathrm{E} \\
+08\end{array}$ & & & & & \\
\hline 87 & 6503 & SA(s)cd & 0.000 & 0.6 & & $\begin{array}{l}3.39 \mathrm{E} \\
+05\end{array}$ & & & & & \\
\hline 88 & 6764 & $\mathrm{SB}(\mathrm{s}) \mathrm{bc}$ & 0.008 & 34.5 & & & & & & & \\
\hline 89 & 7331 & $\mathrm{SA}(\mathrm{s}) \mathrm{b}$ & 0.003 & 11.8 & & \begin{tabular}{|l|}
$3.39 \mathrm{E}$ \\
+05
\end{tabular} & & & & & \\
\hline 90 & 7332 & SO & 0.006 & 24.6 & & $\begin{array}{l}1.30 \mathrm{E} \\
+07\end{array}$ & $\begin{array}{l}5.00 \mathrm{E} \\
+05\end{array}$ & \begin{tabular}{|l|}
$6.00 \mathrm{E}$ \\
+05 \\
\end{tabular} & & & \\
\hline 91 & 7457 & SO & 0.003 & 14.1 & $\bar{A}$ & $\begin{array}{l}3.50 \mathrm{E} \\
+06\end{array}$ & \begin{tabular}{|l}
$1.40 \mathrm{E}$ \\
+06
\end{tabular} & $\begin{array}{l}1.10 \mathrm{E} \\
+06\end{array}$ & $\mathrm{~S}$ & 0.05 & 1 \\
\hline 92 & 7479 & $\mathrm{SB}(\mathrm{s}) \mathrm{C}$ & 0.008 & 34.1 & & & & & & & \\
\hline 93 & $\begin{array}{l}\text { M31 } \\
\text { (224) }\end{array}$ & $\mathrm{SA}(\mathrm{s}) \mathrm{b}$ & 0.0002 & 0.8 & $\bar{A}$ & $\begin{array}{l}4.50 \mathrm{E} \\
+07\end{array}$ & $\begin{array}{l}2.50 \mathrm{E} \\
+07\end{array}$ & \begin{tabular}{|l|}
$4.00 \mathrm{E}$ \\
+07
\end{tabular} & $\mathrm{~S}$ & 2.05 & 41 \\
\hline$\overline{94}$ & $\begin{array}{l}1068 \\
\text { (M77) }\end{array}$ & $\mathrm{Sb}$ & 0.004 & 15.0 & S1.9 & $\begin{array}{l}8.30 \mathrm{E} \\
+06\end{array}$ & $\begin{array}{l}3.00 \mathrm{E} \\
+05\end{array}$ & $\begin{array}{l}3.00 \mathrm{E} \\
+05\end{array}$ & $\bar{M}$ & 0.02 & 2.7 \\
\hline 95 & $\begin{array}{l}3368 \\
\text { (M96) }\end{array}$ & $\begin{array}{c}\mathrm{SAB}(\mathrm{rs}) \\
a b\end{array}$ & 0.003 & 12.9 & & $\begin{array}{l}1.45 \mathrm{E} \\
+07\end{array}$ & & & & & \\
\hline$\overline{96}$ & $\begin{array}{r}3623 \\
(M 65) \\
\end{array}$ & $\begin{array}{c}\mathrm{SAB}(\mathrm{rs}) \\
\mathrm{a} \\
\end{array}$ & 0.003 & 11.6 & & $\begin{array}{l}1.45 \mathrm{E} \\
+08 \\
\end{array}$ & & & & & \\
\hline 97 & $\begin{array}{r}4569 \\
\text { (M90) } \\
\end{array}$ & $\begin{array}{c}\mathrm{SAB}(\mathrm{rs}) \\
\mathrm{ab}\end{array}$ & 0.004 & 18.0 & & $\begin{array}{l}3.80 \mathrm{E} \\
+07 \\
\end{array}$ & & & & & \\
\hline 98 & $\begin{array}{l}4579 \\
\text { (M58) }\end{array}$ & $\begin{array}{c}\mathrm{SAB}(\mathrm{rs}) \\
b\end{array}$ & 0.004 & 18.0 & & $\begin{array}{l}7.08 \mathrm{E} \\
+07\end{array}$ & & & & & \\
\hline 99 & $\begin{array}{l}5194 \\
\text { (M51) }\end{array}$ & $\begin{array}{l}\text { SA(s)bc; } \\
\text { p. }\end{array}$ & 0.002 & 9.0 & & $\begin{array}{l}7.94 \mathrm{E} \\
+06\end{array}$ & & & & & \\
\hline 100 & IC1218 & S? & 0.004 & 15.9 & & & & & & & \\
\hline 101 & M81 & $\mathrm{Sb}$ & 0.001 & 3.9 & & $\begin{array}{l}7.60 \mathrm{E} \\
+07\end{array}$ & $\begin{array}{l}1.10 \mathrm{E} \\
+07\end{array}$ & \begin{tabular}{|l|}
$2.20 \mathrm{E}$ \\
+07
\end{tabular} & $G$ & 0.63 & 13 \\
\hline 102 & MW & Sbbc & $\begin{array}{c}0.00000 \\
2\end{array}$ & 0.0 & Normal & $\begin{array}{l}7.60 \mathrm{E} \\
+0706\end{array}$ & $\begin{array}{l}6.00 \mathrm{E} \\
+\end{array}$ & $\begin{array}{l}6.00 \mathrm{E} \\
+\end{array}$ & S & 42.9 & 171 \\
\hline
\end{tabular}




\begin{tabular}{|c|c|c|c|c|c|c|c|c|c|c|}
\hline \multicolumn{11}{|c|}{ TABLA 2: GALAXIAS ESPIRALES (columnas 13 a 22)" } \\
\hline No. & $\sigma_{c}, \mathrm{~km} / \mathrm{s}$ & $\begin{array}{l}\text { Error en } \\
\sigma_{\mathrm{c}}( \pm)\end{array}$ & $\mathrm{Re}, \mathrm{kpc}$ & $\begin{array}{l}\text { Error Re } \\
\qquad( \pm)\end{array}$ & Lfir, Lsol & $\begin{array}{c}\text { L bulbo } \\
\text { Lsol }\end{array}$ & $\begin{array}{c}\text { Mbul, } \\
10^{\circ} \\
\text { Msol }\end{array}$ & $\begin{array}{l}\text { Error } \\
\text { Mbul, } \\
10^{\circ}( \pm)\end{array}$ & $\begin{array}{c}\text { Gamma } \\
=\mathrm{M} / \mathrm{L} \\
\text { (Msol/ } \\
\text { Lsol) }\end{array}$ & $\begin{array}{l}\text { Banda } \\
\text { en que } \\
\text { se } \\
\text { mide } \\
\text { gamma }\end{array}$ \\
\hline (1) & (13) & (14) & (15) & $(16)$ & (17) & (18) & (19) & (20) & (21) & $(22)$ \\
\hline 51 & & & & & $\begin{array}{l}7.94 \mathrm{E}+ \\
09\end{array}$ & & & & & \\
\hline 52 & & & & & $\begin{array}{l}2.00 \mathrm{E}+ \\
07\end{array}$ & & & & & \\
\hline 53 & & & & & \begin{tabular}{|l|}
$1.26 \mathrm{E}+$ \\
10 \\
\end{tabular} & & & & & \\
\hline 54 & & & & & \begin{tabular}{|l|}
$3.98 \mathrm{E}+$ \\
10
\end{tabular} & & & & & \\
\hline 55 & & & & & \begin{tabular}{|l}
$3.16 \mathrm{E}+$ \\
10 \\
\end{tabular} & & & & & \\
\hline 56 & 205 & & 1.2 & 0.3 & \begin{tabular}{|l}
$3.67 \mathrm{E}+$ \\
07 \\
\end{tabular} & \begin{tabular}{|l|}
$1.20 \mathrm{E}$ \\
+10 \\
\end{tabular} & 34 & 9 & 6.6 & V \\
\hline 57 & & & & & \begin{tabular}{|l|}
$7.94 \mathrm{E}+$ \\
09
\end{tabular} & & & & & \\
\hline 58 & & & & & & & & & & \\
\hline 59 & 140 & & 0.3 & 0.08 & \begin{tabular}{|l|}
$6.62 \mathrm{E}+$ \\
07 \\
\end{tabular} & \begin{tabular}{|l|}
$1.30 \mathrm{E}$ \\
+09 \\
\end{tabular} & 4.4 & 1.2 & & \\
\hline 60 & 165 & & & & \begin{tabular}{|l}
$2.51 \mathrm{E}+$ \\
08 \\
\end{tabular} & \begin{tabular}{|l|}
$2.90 \mathrm{E}$ \\
+09 \\
\end{tabular} & 64 & & & \\
\hline 61 & & & & & \begin{tabular}{|l|}
$2.00 \mathrm{E}+$ \\
10 \\
\end{tabular} & & & & & \\
\hline 62 & 230 & & 4.7 & 1.2 & \begin{tabular}{|l}
$2.66 \mathrm{E}+$ \\
07 \\
\end{tabular} & $\begin{array}{l}1.70 \mathrm{E} \\
+10 \\
\end{array}$ & 170 & 50 & 7 & V \\
\hline 63 & & & & & $\begin{array}{l}1.00 \mathrm{E}+ \\
09\end{array}$ & & & & & \\
\hline 64 & 205 & & 1.3 & 0.3 & $\begin{array}{l}1.74 \mathrm{E}+ \\
09\end{array}$ & $\begin{array}{l}1.70 \mathrm{E} \\
+10 \\
\end{array}$ & 39 & 10 & 3.7 & $\mathrm{R}$ \\
\hline
\end{tabular}

${ }^{11}$ (13) dispersión central de velocidades, en km/s; (14) cota de error en la medición de sigma, en km/s; (15) Re, radio efectivo del bulbo, en kpc; (16) cotas mínima y máxima de error en el cálculo de Re; (17) luminosidad en lejano infrarrojo, en luminosidades solares; (18) luminosidad del bulbo, en luminosidades solares; (19) Mbul, masa del bulbo, en miles de millones de masas solares; (20) cotas de error en el cálculo de Mbul; (21) Gamma, relación masa-luminosidad de toda la galaxia, en masas solares sobre luminosidades solares; (22) banda en la que se mide el valor de gamma: V es luz visible, R es rojo, I es infrarrojo.

Las referencias corresponden al siguiente código de colores, para las 3 tablas:

\begin{tabular}{|cc|l|l|l|}
\hline NED & HFS97 & Ferrarese \& Merrit 2000 & Haring \& Rix 2004 & Wu\& Ca0 2005 \\
\hline Satyapal + 2004 & Gebhardt +2000 & Marconi \& Hunt 2003 & Dudik+2005 & Gallo + 2008 \\
\hline
\end{tabular}




\begin{tabular}{|c|c|c|c|c|c|c|c|c|c|c|}
\hline \multicolumn{11}{|c|}{ TABLA 2: GALAXIAS ESPIRALES (columnas 13 a 22) } \\
\hline No. & $\sigma_{c}, \mathrm{~km} / \mathrm{s}$ & $\mid \begin{array}{l}\text { Error en } \\
\bar{\sigma}_{\mathrm{c}}( \pm)\end{array}$ & $\mathrm{Re}, \mathrm{kpc}$ & $\begin{array}{l}\text { Error Re } \\
\qquad( \pm)\end{array}$ & Lfir, Lsol & $\begin{array}{c}\text { L bulbo } \\
\text { Lsol }\end{array}$ & $\begin{array}{c}\text { Mbul, } \\
10^{\circ} \\
\text { Msol }\end{array}$ & $\begin{array}{c}\text { Error } \\
\text { Mbul, } \\
10^{\circ}( \pm)\end{array}$ & $\begin{array}{c}\text { Gamma } \\
=\mathrm{M} / \mathrm{L} \\
\text { (Msol/ } \\
\text { Lsol) }\end{array}$ & \begin{tabular}{|} 
Banda \\
en que \\
se \\
mide \\
gamma
\end{tabular} \\
\hline (1) & (13) & (14) & (15) & $(16)$ & $(17)$ & (18) & (19) & $(20)$ & $(21)$ & $(22)$ \\
\hline 65 & 143 & & 0.5 & 0.12 & \begin{tabular}{|l|}
$4.27 \mathrm{E}+$ \\
07
\end{tabular} & \begin{tabular}{|l|}
$7.10 \mathrm{E}$ \\
+09
\end{tabular} & 7 & 1.9 & 2.8 & V \\
\hline 66 & & & & & $\begin{array}{l}1.58 \mathrm{E}+ \\
09\end{array}$ & & & & & \\
\hline 67 & & & & & $\begin{array}{l}1.00 \mathrm{E}+ \\
10\end{array}$ & & & & & \\
\hline 68 & 130 & & 0.9 & 0.23 & \begin{tabular}{|l|}
$3.13 E+$ \\
09
\end{tabular} & \begin{tabular}{|l|}
$1.20 \mathrm{E}$ \\
+09 \\
\end{tabular} & 11 & 3 & & \\
\hline 69 & & & & & $\begin{array}{l}1.00 \mathrm{E}+ \\
09\end{array}$ & & & & & \\
\hline 70 & 225 & & 0.3 & 0.07 & & \begin{tabular}{|l|}
$1.90 \mathrm{E}$ \\
+09
\end{tabular} & 10 & 3 & 6.3 & $\mathrm{~T}$ \\
\hline 71 & & & & & \begin{tabular}{|l|}
$2.00 \mathrm{E}+$ \\
08
\end{tabular} & & & & & \\
\hline 72 & & & & & $\begin{array}{l}3.98 \mathrm{E}+ \\
09\end{array}$ & & & & & \\
\hline 73 & 186 & & 15 & 4 & $\begin{array}{l}1.08 \mathrm{E}+ \\
09\end{array}$ & \begin{tabular}{|l|}
$7.00 \mathrm{E}$ \\
+09 \\
\end{tabular} & 360 & 100 & & \\
\hline 74 & & & & & $\begin{array}{l}2.51 \mathrm{E}+ \\
10\end{array}$ & & & & & \\
\hline 75 & 240 & & 5.1 & 1.3 & $\begin{array}{l}1.46 \mathrm{E}+ \\
09\end{array}$ & \begin{tabular}{|l|}
$\begin{array}{l}4.40 \mathrm{E} \\
+10\end{array}$ \\
\end{tabular} & 200 & 50 & 6.1 & $\mathrm{~V}$ \\
\hline 76 & 152 & & 1.6 & 0.4 & \begin{tabular}{|l}
$8.76 \mathrm{E}+$ \\
08
\end{tabular} & \begin{tabular}{|l|}
$2.70 \mathrm{E}$ \\
+10
\end{tabular} & 26 & 7 & & \\
\hline 77 & & & & & \begin{tabular}{|l}
$1.26 \mathrm{E}+$ \\
09
\end{tabular} & & & & & \\
\hline 78 & & & & & \begin{tabular}{|l|}
$2.51 \mathrm{E}+$ \\
10
\end{tabular} & & & & & \\
\hline 79 & & & & & $\begin{array}{l}6.31 \mathrm{E}+ \\
08\end{array}$ & & & & & \\
\hline 80 & & & & & \begin{tabular}{|l}
$6.31 \mathrm{E}+$ \\
09
\end{tabular} & & & & & \\
\hline 81 & 150 & & 3.6 & 0.9 & & \begin{tabular}{|l|}
$3.20 \mathrm{E}$ \\
+10 \\
\end{tabular} & 56 & 15 & & \\
\hline 82 & 190 & & 9.7 & 2.4 & & \begin{tabular}{|l|}
$3.20 \mathrm{E}$ \\
+10 \\
\end{tabular} & 240 & 90 & & \\
\hline 83 & & & & & $\begin{array}{l}1.26 \mathrm{E}+ \\
10\end{array}$ & & & & & \\
\hline
\end{tabular}




\begin{tabular}{|c|c|c|c|c|c|c|c|c|c|c|}
\hline \multicolumn{11}{|c|}{ TABLA 2: GALAXIAS ESPIRALES (columnas 13 a 22) } \\
\hline No. & $\sigma_{c}, \mathrm{~km} / \mathrm{s}$ & $\begin{array}{l}\text { Error en } \\
\bar{\sigma}_{\mathrm{c}}( \pm)\end{array}$ & $\mathrm{Re}, \mathrm{kpc}$ & $\begin{array}{c}\text { Error Re } \\
( \pm)\end{array}$ & Lfir, Lsol & $\begin{array}{c}\text { L bulbo } \\
\text { Lsol }\end{array}$ & $\begin{array}{c}\text { Mbul, } \\
10^{\circ} \\
\text { Msol }\end{array}$ & $\begin{array}{l}\text { Error } \\
\text { Mbul, } \\
10^{\circ}( \pm)\end{array}$ & $\begin{array}{c}\text { Gamma } \\
=\mathrm{M} / \mathrm{L} \\
\text { (Msol/ } \\
\text { Lsol) }\end{array}$ & $\begin{array}{c}\text { Banda } \\
\text { en que } \\
\text { se } \\
\text { mide } \\
\text { gamma }\end{array}$ \\
\hline (1) & (13) & (14) & (15) & (16) & (17) & (18) & (19) & $\begin{array}{l}(20) \\
\end{array}$ & (21) & $(22)$ \\
\hline 84 & & & & & $\begin{array}{l}7.94 \mathrm{E}+ \\
09\end{array}$ & & & & & \\
\hline 85 & & & & & $\begin{array}{l}1.26 \mathrm{E}+ \\
10\end{array}$ & & & & & \\
\hline 86 & & & & & $2.51 \mathrm{E}+$ & & & & & \\
\hline 87 & & & & & $\begin{array}{l}6.31 \mathrm{E}+ \\
06\end{array}$ & & & & & \\
\hline 88 & & & & & $\begin{array}{l}1.00 \mathrm{E}+ \\
10\end{array}$ & & & & & \\
\hline 89 & & & & & $\begin{array}{l}6.31 \mathrm{E}+ \\
06\end{array}$ & & & & & \\
\hline 90 & 122 & & & & & $\begin{array}{l}7.90 \mathrm{E} \\
+09\end{array}$ & 15 & & 1.9 & V \\
\hline 91 & 67 & & 4.8 & 3.5 & $\begin{array}{l}5.40 \mathrm{E}+ \\
07\end{array}$ & \begin{tabular}{|l|}
$2.10 \mathrm{E}$ \\
+09
\end{tabular} & 15 & 11 & 3.4 & $\mathrm{~V}$ \\
\hline 92 & & & & & $\begin{array}{l}2.00 \mathrm{E}+ \\
10\end{array}$ & & & & & \\
\hline 93 & 160 & & 1 & 0.3 & $\begin{array}{l}1.25 \mathrm{E}+ \\
09\end{array}$ & $\begin{array}{l}7.30 \mathrm{E} \\
+09\end{array}$ & 19 & 5 & 5.1 & $\mathrm{~V}$ \\
\hline 94 & 151 & & 3.1 & 0.8 & $\begin{array}{l}7.13 \mathrm{E}+ \\
10\end{array}$ & \begin{tabular}{|l|}
$1.50 \mathrm{E}$ \\
+11 \\
\end{tabular} & 50 & 14 & 0.15 & $R$ \\
\hline 95 & & & & & $\begin{array}{l}2.51 \mathrm{E}+ \\
09\end{array}$ & & & & & \\
\hline 96 & & & & & $\begin{array}{l}1.00 \mathrm{E}+ \\
09\end{array}$ & & & & & \\
\hline 97 & & & & & $\begin{array}{l}5.01 \mathrm{E}+ \\
09\end{array}$ & & & & & \\
\hline 98 & & & & & $\begin{array}{l}3.16 \mathrm{E}+ \\
09\end{array}$ & & & & & \\
\hline 99 & & & & & $\begin{array}{l}6.31 \mathrm{E}+ \\
09\end{array}$ & & & & & \\
\hline 100 & & & & & $\begin{array}{l}3.16 \mathrm{E}+ \\
08\end{array}$ & & & & & \\
\hline 101 & 165 & & 3.4 & 0.9 & & & 64 & 18 & & \\
\hline 102 & 103 & & 0.7 & 0.2 & & \begin{tabular}{|l|}
$1.80 \mathrm{E}$ \\
+09
\end{tabular} & 5200 & 2500 & & \\
\hline
\end{tabular}




\begin{tabular}{|c|c|c|c|c|c|c|c|c|c|c|c|}
\hline \multicolumn{12}{|c|}{ TABLA 3: GALAXIAS PECULIARES (ACTIVAS E IRREGULARES) ${ }^{12}$} \\
\hline No. & Nombre & \begin{tabular}{|c} 
Tipo \\
Hubble
\end{tabular} & $\begin{array}{c}\mathrm{Z} \\
\text { Estimado } \\
\text { con Ho=70 } \\
\mathrm{Km} / \mathrm{s} / \mathrm{mpc}\end{array}$ & $\begin{array}{l}\mathrm{D}, \mathrm{Mpc} \\
(\mathrm{HO}=70)\end{array}$ & $\begin{array}{c}\text { Tipo } \\
\text { nuclear }\end{array}$ & $\begin{array}{l}\mathrm{M}_{\mathrm{BH}}, \\
\text { Msol }\end{array}$ & $\begin{array}{l}\text { Error } \\
\mathrm{M}_{\mathrm{BH}},-\end{array}$ & $\begin{array}{l}\text { Error } \\
\mathrm{M}_{\mathrm{BH}},+\end{array}$ & Ref & $\begin{array}{c}\mathrm{R}_{\mathrm{BH}}, \\
\operatorname{arcsec}\end{array}$ & Nres \\
\hline (1) & (2) & (3) & (4) & (5) & (6) & (7) & (8) & (9) & (10) & (11) & $(12)$ \\
\hline 103 & 205 (M110) & & 0.0002 & 0.079 & & $\begin{array}{c}9.33 \mathrm{E}+ \\
04\end{array}$ & & & & & \\
\hline 104 & $\begin{array}{c}863 \text { (Mrk } \\
590)\end{array}$ & & 0.025 & 117.0 & & $\begin{array}{c}1.78 \mathrm{E}+ \\
07\end{array}$ & & & & & \\
\hline 105 & 3227 & & 0.005 & 22.1 & & $\begin{array}{c}3.89 \mathrm{E}+ \\
07\end{array}$ & & & & & \\
\hline 106 & 3516 & & 0.009 & 41.7 & & $\begin{array}{c}2.29 \mathrm{E}+ \\
07\end{array}$ & & & & & \\
\hline 107 & 3783 & & 0.009 & 41.3 & & $\begin{array}{c}9.33 \mathrm{E}+ \\
06\end{array}$ & & & & & \\
\hline 108 & 4051 & & 0.004 & 18.2 & & $\begin{array}{c}1.29 \mathrm{E}+ \\
06\end{array}$ & & & & & \\
\hline 109 & 4151 & & 0.005 & 21.8 & & $\begin{array}{c}1.51 \mathrm{E}+ \\
07\end{array}$ & & & & & \\
\hline 110 & 4593 & & 0.009 & 42.3 & & $\begin{array}{c}7.94 \mathrm{E}+ \\
06\end{array}$ & & & & & \\
\hline 111 & 4945 & & 0.001 & 4.5 & & $\begin{array}{c}1.10 \mathrm{E}+ \\
06\end{array}$ & & & & & \\
\hline 112 & 5548 & & 0.016 & 75.2 & & $\begin{array}{c}1.23 \mathrm{E}+ \\
08\end{array}$ & & & & & \\
\hline 113 & 7469 & & 0.016 & 71.4 & & $\begin{array}{c}6.46 \mathrm{E}+ \\
06\end{array}$ & & & & & \\
\hline 114 & $3 Z W 35$ & & 0.026 & 120.6 & & & & & & & $\begin{array}{c}2.00 \mathrm{E} \\
+11\end{array}$ \\
\hline 115 & $\begin{array}{c}\text { IRAS } \\
01173+ \\
1405 \\
\end{array}$ & & 0.029 & 133.8 & & & & & & & $\begin{array}{c}\text { TII } \\
\begin{array}{c}2.00 \mathrm{E} \\
+11\end{array}\end{array}$ \\
\hline 116 & $\begin{array}{l}\text { Mrk 1506 } \\
\text { (3C 120) }\end{array}$ & & 0.032 & 147.6 & & $\begin{array}{c}2.29 \mathrm{E}+ \\
07\end{array}$ & & & & & \\
\hline
\end{tabular}

12(1) número asignado; (2) nombre más común de la galaxia; (3) tipo de galaxia según la clasificación de Hubble; (4) corrimiento al rojo (z) en base a una constante de Hubble $\mathrm{H}_{0}=70 \mathrm{~km} \mathrm{~s}^{-1} \mathrm{Mpc}^{-1}$; (5) distancia a la galaxia, en Mega pársecs; (6) tipo del núcleo, según el artículo de Ho, Phillipenko \& Sargent de 1997; (7) masa calculada del agujero negro súper masivo, en masas solares; (8) cota mínima de error para $\mathrm{M}_{\mathrm{BH}}$, en masas solares; (9) cota máxima de error para $\mathrm{M}_{\text {вн, }}$, en masas solares; (10) método empleado para el cálculo de $\mathrm{M}_{в н}$ : $g$ es gas, $\mathrm{s}$ es estrellas, $m$ es máser; (11) $R_{B H}$, radio de influencia del ANS, en segundos de arco; (12) luminosidad en lejano infrarrojo, en luminosidades solares.

Las referencias corresponden al siguiente código de colores, para las 3 tablas:

\begin{tabular}{|cc|l|l|l|}
\hline NED & HFS97 & Ferrarese \& Merrit 2000 & Haring \& Rix 2004 & Wu\& Cao 2005 \\
\hline Satyapal + 2004 & Gebhardt +2000 & Marconi \& Hunt 2003 & Dudik+ 2005 & Gallo + 2008 \\
\hline
\end{tabular}




\begin{tabular}{|c|c|c|c|c|c|c|c|c|c|c|c|}
\hline \multicolumn{12}{|c|}{ TABLA 3: GALAXIAS PECULIARES (ACTIVAS E IRREGULARES) } \\
\hline No. & Nombre & $\begin{array}{l}\text { Tipo } \\
\text { Hubble }\end{array}$ & $\begin{array}{c}\mathrm{Z} \\
\text { Estimado } \\
\text { con Ho=70 } \\
\mathrm{Km} / \mathrm{s} / \mathrm{mpc}\end{array}$ & $\begin{array}{c}\mathrm{D}, \mathrm{Mpc} \\
(\mathrm{HO}=70)\end{array}$ & $\begin{array}{c}\text { Tipo } \\
\text { nuclear }\end{array}$ & $\begin{array}{l}\mathrm{M}_{\mathrm{BH}}, \\
\text { Msol }\end{array}$ & $\begin{array}{l}\text { Error } \\
\mathrm{M}_{\mathrm{BH}} \text {, - }\end{array}$ & $\begin{array}{l}\text { Error } \\
\mathrm{M}_{\mathrm{BH}},+\end{array}$ & Ref & $\begin{array}{c}\mathrm{R}_{\mathrm{BH}}, \\
\operatorname{arcsec}\end{array}$ & Nres \\
\hline (1) & $(2)$ & (3) & $(4)$ & (5) & (6) & (7) & $(8)$ & (9) & $(10)$ & (11) & $(12)$ \\
\hline 117 & Mrk 110 & & 0.034 & 158.3 & & $\begin{array}{c}5.50 \mathrm{E}+ \\
06\end{array}$ & & & & & \\
\hline 118 & $\begin{array}{c}\text { Mrk 279 } \\
\text { (UGC 8823) }\end{array}$ & & 0.030 & 135.6 & & $\begin{array}{c}4.17 \mathrm{E}+ \\
07\end{array}$ & & & & & \\
\hline 119 & Mrk 335 & & 0.025 & 114.2 & & $\begin{array}{c}6.17 \mathrm{E}+ \\
06\end{array}$ & & & & & \\
\hline 120 & Mrk 509 & & 0.034 & 154.1 & & $\begin{array}{c}5.75 \mathrm{E}+ \\
07\end{array}$ & & & & & \\
\hline 121 & \begin{tabular}{|c|} 
Mrk 817 \\
(UGC 9412) \\
\end{tabular} & & 0.031 & 140.4 & & \begin{tabular}{|c}
$4.37 \mathrm{E}+$ \\
07 \\
\end{tabular} & & & & & \\
\hline 122 & Fairall 9 & & 0.047 & 214.1 & & $\begin{array}{c}7.94 \mathrm{E}+ \\
07\end{array}$ & & & & & \\
\hline 123 & IC $4329 \mathrm{~A}$ & & 0.015 & 70.2 & & $\begin{array}{c}4.90 \mathrm{E}+ \\
06\end{array}$ & & & & & \\
\hline 124 & $\begin{array}{c}\text { Mrk 79 } \\
\text { (UGC 3973) }\end{array}$ & & 0.021 & 97.8 & & $\begin{array}{c}5.13 \mathrm{E}+ \\
07\end{array}$ & & & & & \\
\hline 125 & \begin{tabular}{|c} 
VII w 838 \\
(3C 890.3)
\end{tabular} & & 0.056 & 258.4 & & $\begin{array}{c}3.39 \mathrm{E}+ \\
08\end{array}$ & & & & & \\
\hline 126 & $\begin{array}{l}\text { Mrk 1095 } \\
\text { (Ark 120) }\end{array}$ & & 0.031 & 144.2 & & $\begin{array}{c}1.82 \mathrm{E}+ \\
08\end{array}$ & & & & & \\
\hline 127 & Arp 102B & & 0.023 & 106.8 & & $\begin{array}{c}2.19 \mathrm{E}+ \\
08\end{array}$ & & & & & \\
\hline 128 & $\begin{array}{l}\text { Mrk } 1095 \\
\text { (Ark 120) }\end{array}$ & & 0.031 & 144.2 & & \begin{tabular}{|c}
$1.82 \mathrm{E}+$ \\
08
\end{tabular} & & & & & \\
\hline 129 & Cicinus & & 0.001 & 4.3 & & $\begin{array}{c}1.29 \mathrm{E}+ \\
06\end{array}$ & & & & & \\
\hline 130 & 4750 & $\begin{array}{l}\text { (R)SA } \\
\text { (rs)ab }\end{array}$ & 0.005 & 23.7 & & & & & & & $\begin{array}{c}5.01 \mathrm{E} \\
+09 \\
\end{array}$ \\
\hline 131 & 7465 & $\begin{array}{c}\text { (R')SB } \\
\text { (s)0 }\end{array}$ & 0.006 & 28.2 & & $\begin{array}{c}3.89 \mathrm{E}+ \\
07\end{array}$ & & & & & $\begin{array}{c}5.01 \mathrm{E} \\
+09\end{array}$ \\
\hline 132 & $\begin{array}{c}\text { SDSS } \\
1346+2650\end{array}$ & $\begin{array}{c}\mathrm{CD} ; \mathrm{SO} \\
?\end{array}$ & 0.0059 & 271.1 & & $\begin{array}{c}1.17 \mathrm{E}+ \\
09\end{array}$ & & & & & $\begin{array}{c}3.98 \mathrm{E} \\
+10\end{array}$ \\
\hline 133 & Mrk 266NE & $\begin{array}{l}\text { Compa } \\
\text { ct:pcc }\end{array}$ & 0.026 & 120.2 & & & & & & & $\begin{array}{c}1.58 \mathrm{E} \\
+11\end{array}$ \\
\hline 134 & $\begin{array}{c}\text { An0248+ } \\
43 A\end{array}$ & Gpair & 0.048 & 219.9 & & & & & & & $\begin{array}{c}3.16 \mathrm{E} \\
+11\end{array}$ \\
\hline 135 & $\begin{array}{c}\text { SDSS } \\
0248+4302\end{array}$ & Gpair & 0.048 & 219.9 & & & & & & & $\begin{array}{c}3.16 \mathrm{E} \\
+11\end{array}$ \\
\hline 136 & 6240 & $10: ; p$. & 0.023 & 104.9 & & $\begin{array}{c}1.41 \mathrm{E}+ \\
09\end{array}$ & & & & & $\begin{array}{c}2.00 \mathrm{E} \\
+11\end{array}$ \\
\hline 137 & UGC 8387 & $\mathrm{IM} ; \mathrm{pcc}$ & 0.022 & 100.1 & & & & & & & $\begin{array}{c}2.00 \mathrm{E} \\
+11\end{array}$ \\
\hline 138 & \begin{tabular}{|c} 
IRAS20551 \\
-4250 \\
\end{tabular} & Merger & 0.040 & 183.5 & & & & & & & $\begin{array}{r}5.01 \mathrm{E} \\
+11 \\
\end{array}$ \\
\hline
\end{tabular}




\begin{tabular}{|c|c|c|c|c|c|c|c|c|c|c|c|}
\hline \multicolumn{12}{|c|}{ TABLA 3: GALAXIAS PECULIARES (ACTIVAS E IRREGULARES) } \\
\hline No. & Nombre & $\begin{array}{l}\text { Tipo } \\
\text { Hubble }\end{array}$ & $\begin{array}{c}\mathrm{Z} \\
\text { Estimado } \\
\text { con Ho=70 } \\
\text { Km/s/mpc } \\
\end{array}$ & $\begin{array}{c}\mathrm{D}, \mathrm{Mpc} \\
(\mathrm{HO}=70)\end{array}$ & $\begin{array}{c}\text { Tipo } \\
\text { nuclear }\end{array}$ & $\begin{array}{l}\mathrm{M}_{\mathrm{BH}}, \\
\text { Msol }\end{array}$ & $\begin{array}{l}\text { Error } \\
\mathrm{M}_{\mathrm{BH}}{ }^{-}-\end{array}$ & $\begin{array}{l}\text { Error } \\
\mathrm{M}_{\mathrm{BH}},+\end{array}$ & Ref & $\left|\begin{array}{c}\mathrm{R}_{\mathrm{BH}}, \\
\operatorname{arcsec}\end{array}\right|$ & Nres \\
\hline (1) & $(2)$ & (3) & (4) & $(5)$ & $(6)$ & $(7)$ & (8) & $(9)$ & $(10)$ & (11) & $(12)$ \\
\hline 139 & $\begin{array}{c}\text { IRAS } \\
23128-5919\end{array}$ & Merger & 0.042 & 191.1 & & & & & & & $\begin{array}{c}5.01 \mathrm{E} \\
+11\end{array}$ \\
\hline 140 & \begin{tabular}{|c|} 
SDSS \\
$2055-4250$
\end{tabular} & Merger & 0.040 & 183.5 & & & & & & & $\begin{array}{c}5.01 \mathrm{E} \\
+11\end{array}$ \\
\hline 141 & \begin{tabular}{|c|} 
SDSS \\
$2312-5919$
\end{tabular} & Merger & 0.042 & 191.1 & & & & & & & $\begin{array}{c}5.01 \mathrm{E} \\
+11\end{array}$ \\
\hline 142 & Mrk 273 & $\begin{array}{l}\text { Ring } \\
\text { gal. }\end{array}$ & 0.055 & 161.1 & & $\begin{array}{c}5.50 \mathrm{E}+ \\
07\end{array}$ & & & & & $\begin{array}{c}6.31 \mathrm{E} \\
+11\end{array}$ \\
\hline
\end{tabular}

\section{GRÁFICOS DE CORRELACIONES}

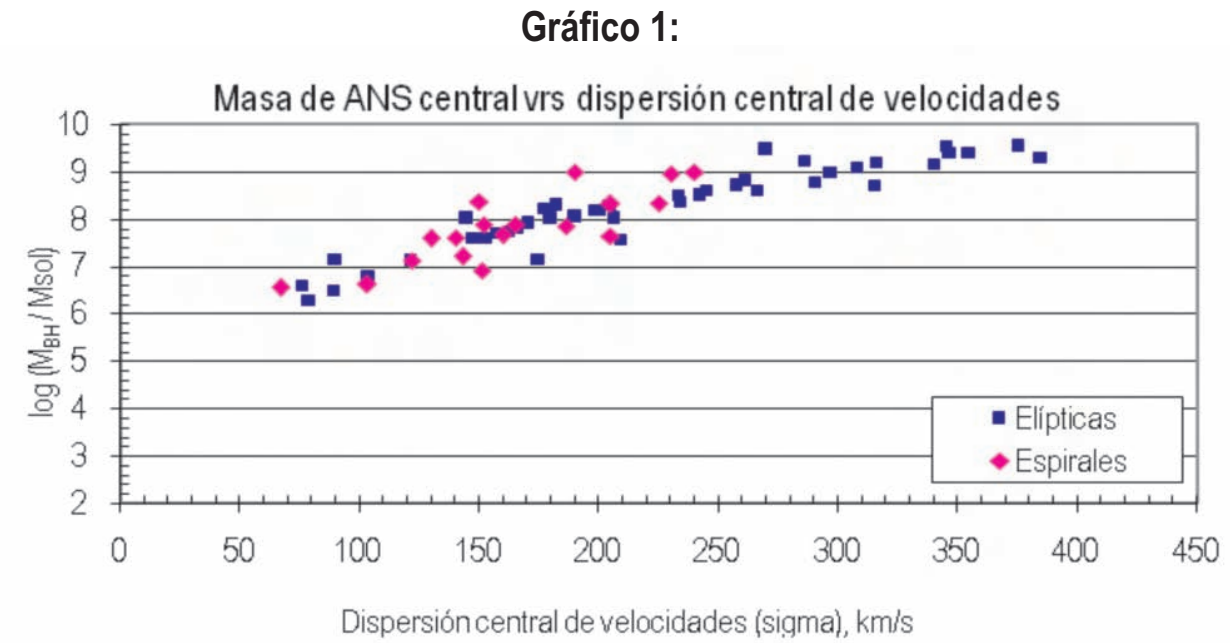

\section{Descripción}

Es bien conocido que la correlación más fuerte con la masa de agujeros negros súper masivos centrales es la dispersión central de velocidades. No obstante, la dispersión es mayor en el caso de las galaxias espirales que en el de las elípticas (nota: no se logró obtener datos de dispersión de velocidad para las galaxias peculiares). 


\section{Gráfico 2:}

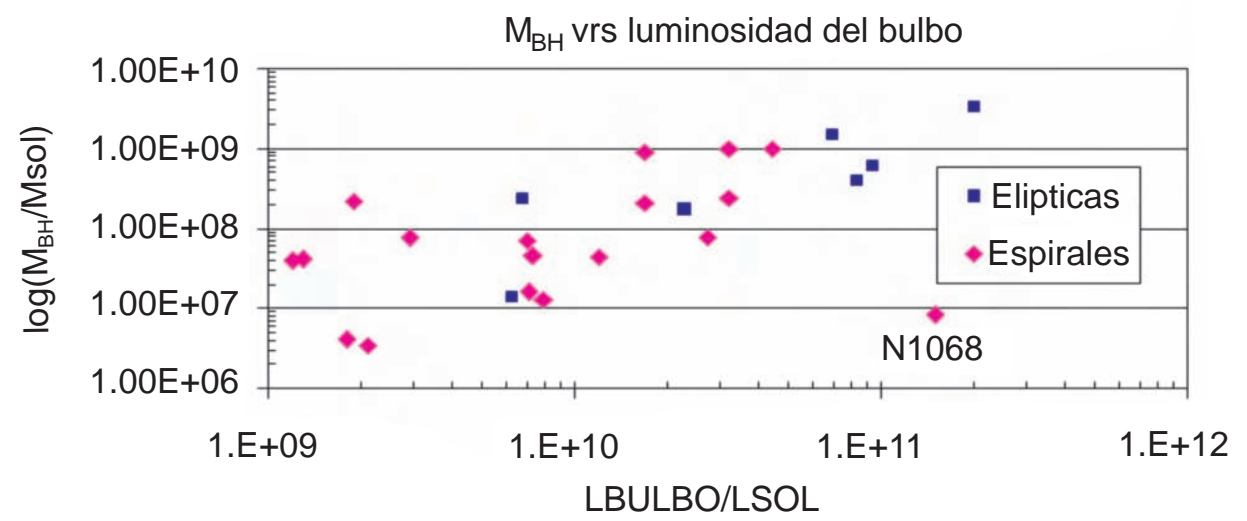

\section{Descripción}

La luminosidad del esferoide anfitrión del ANS también evidencia una correlación fuerte con la masa de ANS. El punto que parece separarse de la tendencia es de la galaxia Seyfert NGC 1068.

\section{Gráfico 3:}

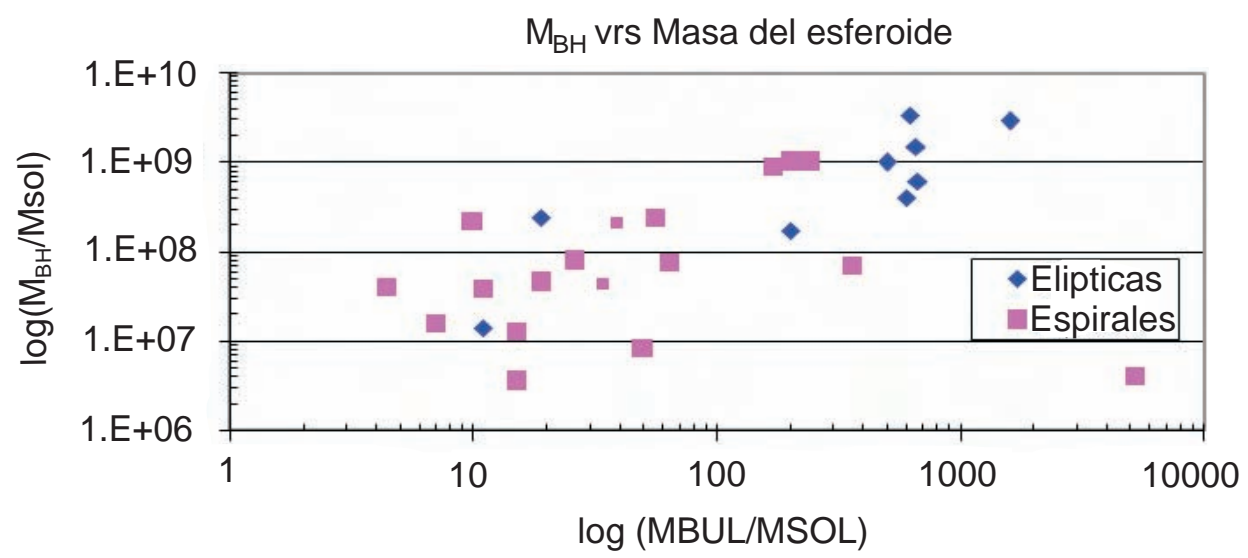

\section{Descripción}

La correlación con la masa del esferoide tiene mayor dispersión que las dos anteriores, especialmente en el caso de las galaxias espirales. 


\section{Gráfico 4:}

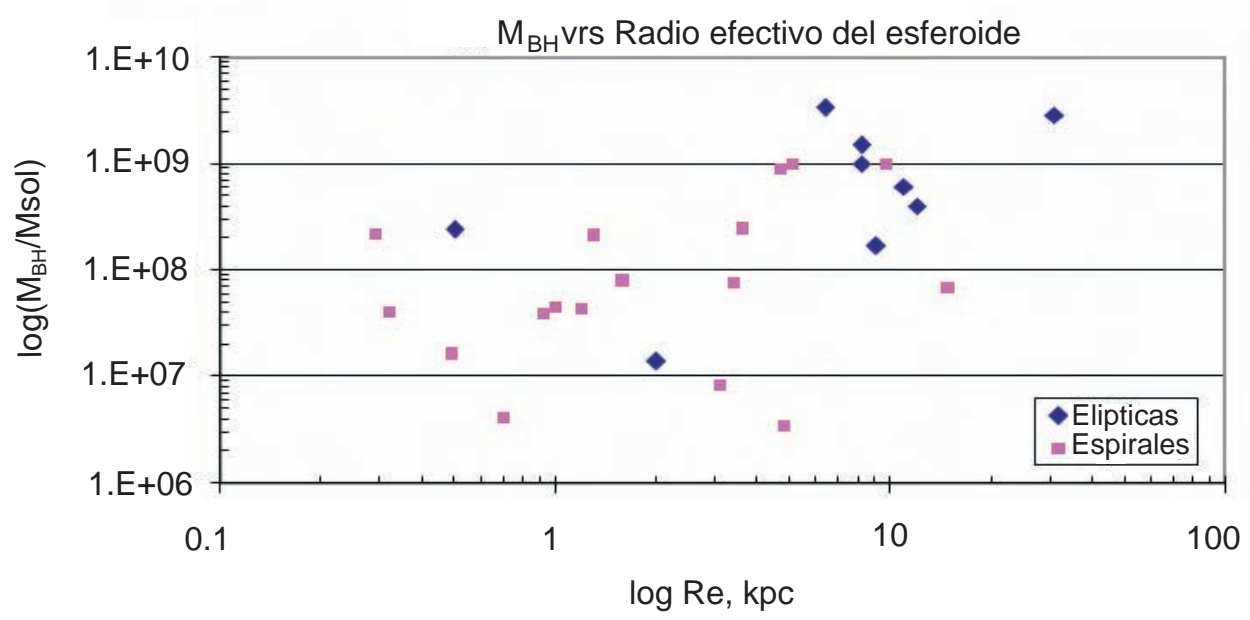

\section{Descripción}

Aquí vemos que la dispersión de la correlación masa de ANS vrs radio efectivo del esferoide es bastante alta.

\section{Gráfico 5:}

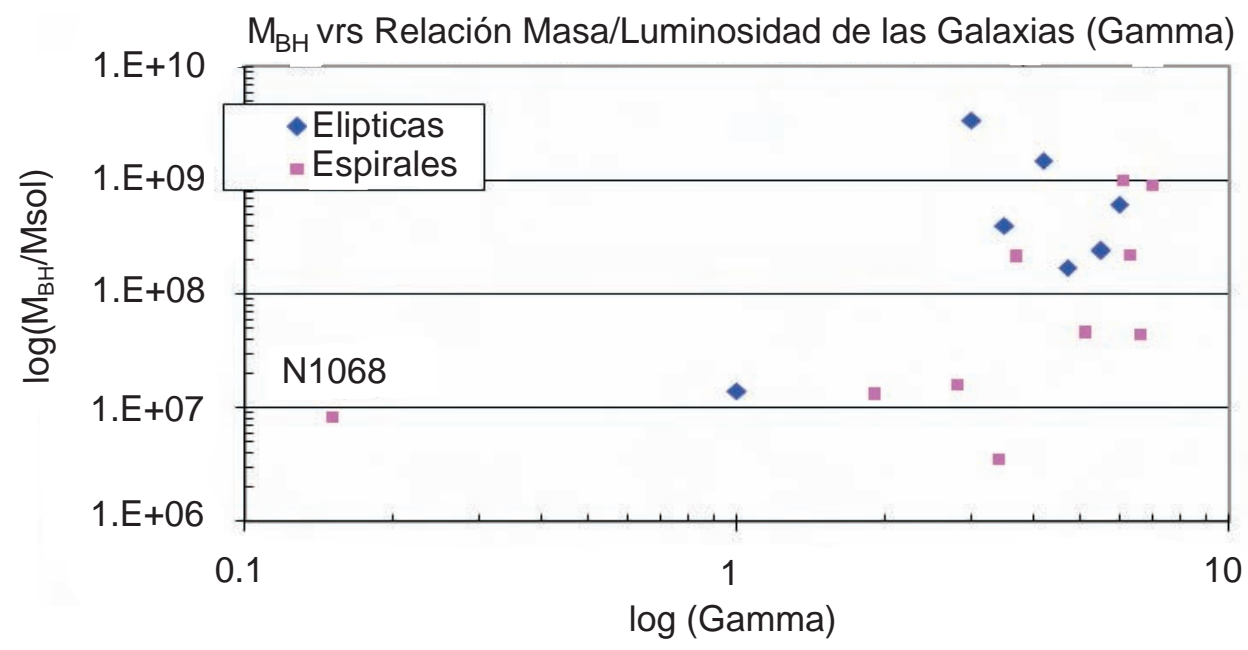

\section{Descripción}

La correlación con la relación Gamma (Masa/Luminosidad totales) es más evidente en el caso de las galaxias espirales, con excepción de la galaxia Seyfert NGC 1068. 


\section{Gráfico 6:}

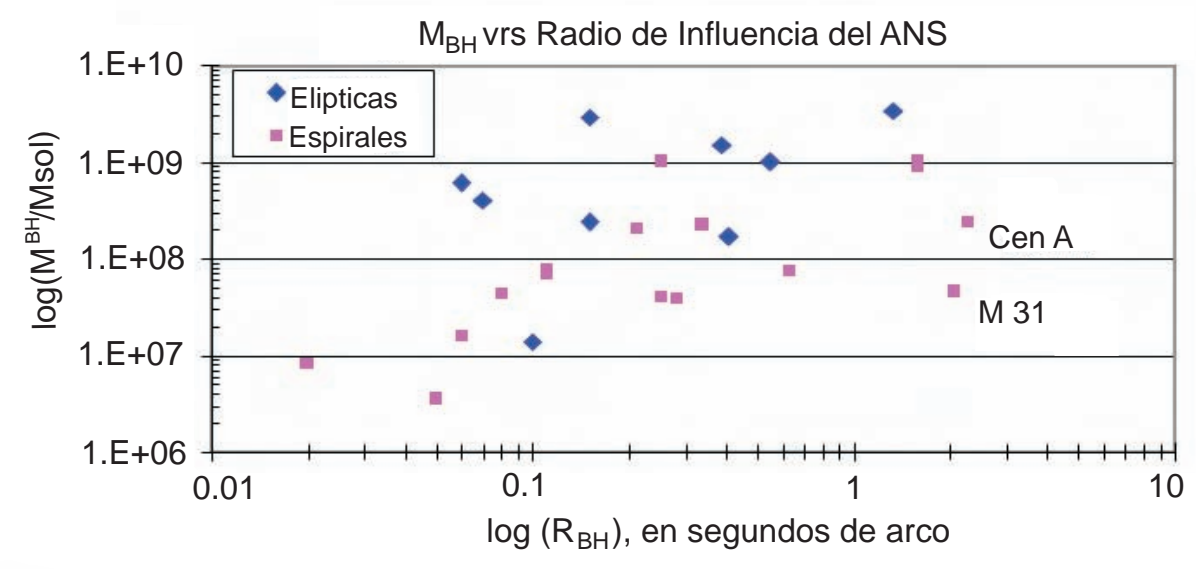

\section{Descripción}

La correlación con el radio de influencia del ANS también existe, aunque las galaxias Centaurus A (una fusión de dos galaxias) y M 31 (Andrómeda) parecen desviarse del resto de puntos.

\section{Gráfico 7:}

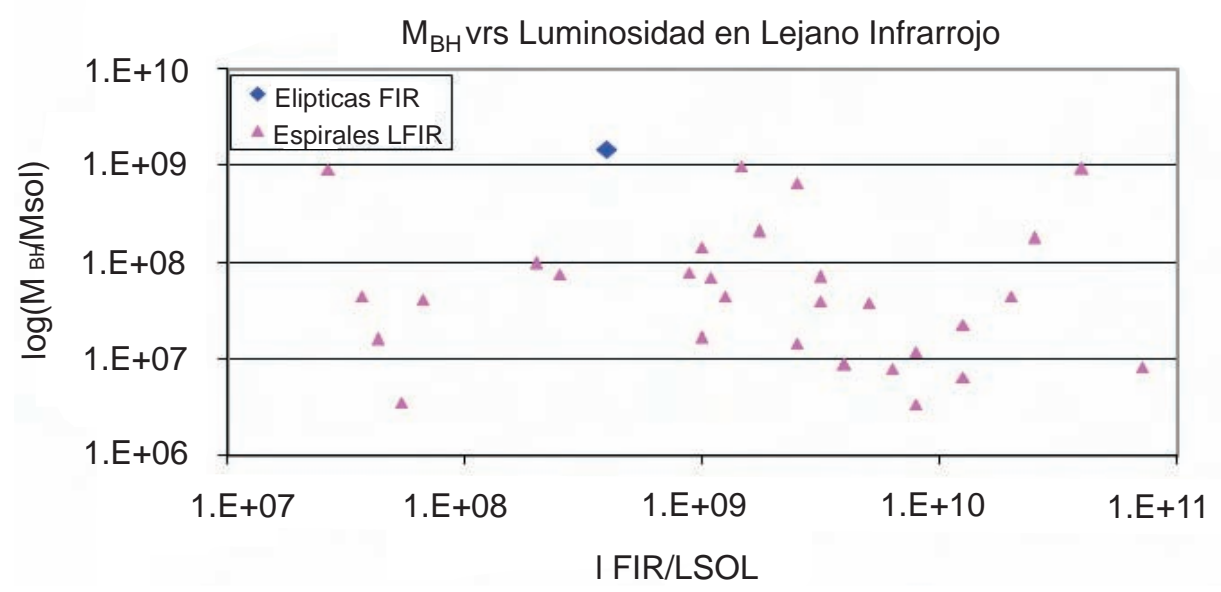

\section{Descripción}

En el caso de la luminosidad total en lejano infrarrojo no se observa correlación con la masa del agujero negro central. Esto parece evidenciar que los discos de las galaxias espirales han seguido un proceso de formación independiente del agujero negro súper masivo y del esferoide. 
Gráfico 8:

$\mathrm{M}_{\mathrm{BH}}$ vrs Corrimiento al Rojo

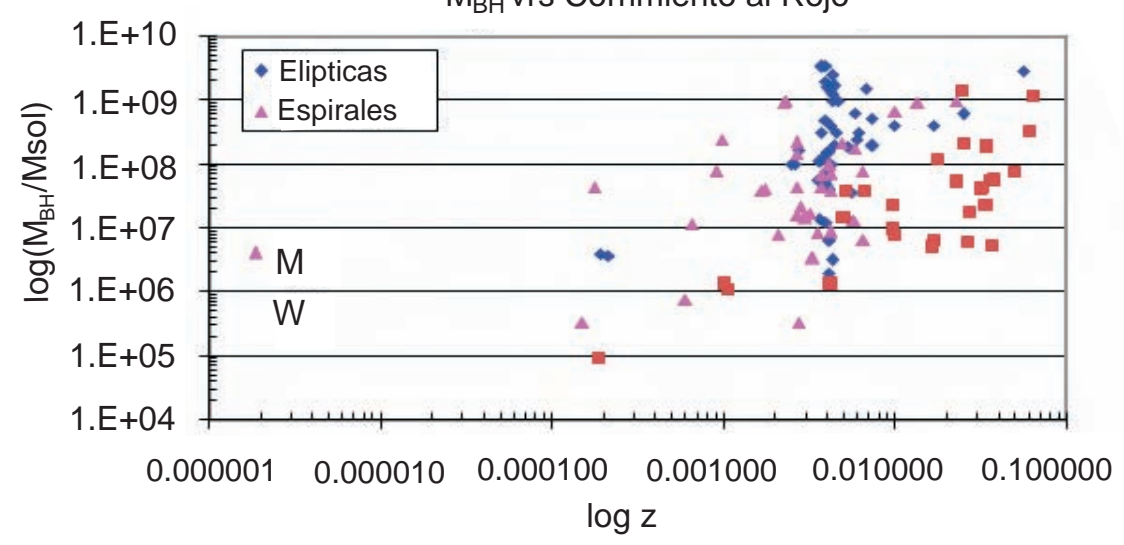

\section{Descripción}

Distribución en el espacio de las correlaciones Mbh- para los tres tipos de galaxias. Las galaxias elípticas están distribuidas entre 0.00019 y 0.056 , pero la mayoría se ubica alrededor de $z=0.004$. Las galaxias espirales están distribuidas entre 0.000002 y 0.023 , donde el valor menor de $z$ corresponde a Nuestra Galaxia. Para las galaxias peculiares (activas en su mayoría), z oscila entre 0.0043 y 0.0633 , lo que las ubica entre las más distantes.

\section{Gráfico 9:}

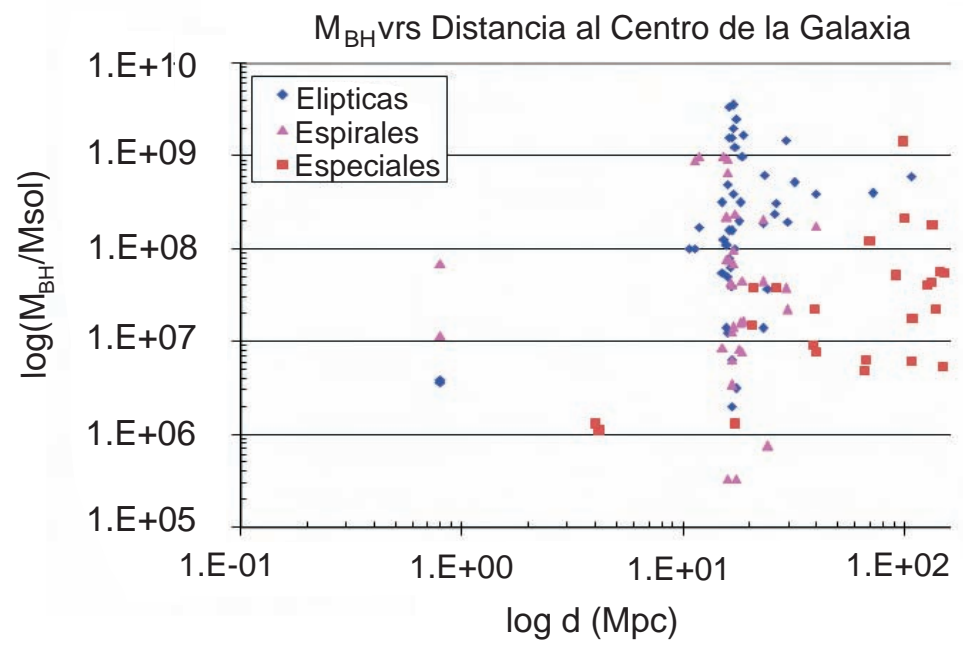

\section{Descripción}

Similar al gráfico anterior, éste muestra la distribución de distancias de las galaxias de la muestra. La mayoría de galaxias elípticas y espirales se encuentran a distancias entre los $15 \mathrm{y}$ $19 \mathrm{Mpc}$. Las galaxias peculiares se encuentran principalmente entre los 16 y los $253 \mathrm{Mpc}$. 
En resumen, entre masa de agujero negro central y dispersión central de velocidades parece ser que la correlación es más fuerte cuando se trata de galaxias espirales que en el caso de galaxias elípticas, contrario a lo que afirman algunos autores. También la correlación de masas de agujeros negros contra luminosidades de los esferoides (bulbos en las espirales o galaxias elípticas) presenta una relación más evidente en el caso de las galaxias espirales que en el de las galaxias elípticas, excepto por el caso de la galaxia Seyfert NGC 1068. Similar situación se presenta en la correlación con la masa del esferoide, pero con mayor dispersión. El radio efectivo del esferoide no presenta correlación evidente con la masa de agujero negro, lo cual no es extraño, pues el radio de influencia del ANS se limita a las regiones centrales; en cambio, a distancias de la proporción del radio del esferoide, el ANS no tiene ninguna influencia. En la relación masaluminosidad total de las galaxias, en general, las elípticas también presentan más dispersión; no obstante, la galaxia más dispersa es la NGC 1068, que es tipo Seyfert (espiral activa). La correlación con el radio de influencia del agujero negro también existe, aunque Centaurus A (galaxia peculiar) y M 31 (Andrómeda) están más separadas del resto de la muestra. La luminosidad total de las galaxias en lejano infrarrojo no presenta una correlación con la masa de agujero negro central.

\section{ANÁLISIS DE MODELOS DE FORMACIÓN YEVOLUCIÓN DE GALAXIAS}

Aquí se relacionan los gráficos de correlaciones con algunos modelos de formación deANSs.

Modelos 1, 2, 3 y 4. Modelos de Volonteri, Lodato \& Natarajan 2008, y Modelo de Estrellas de Población III.

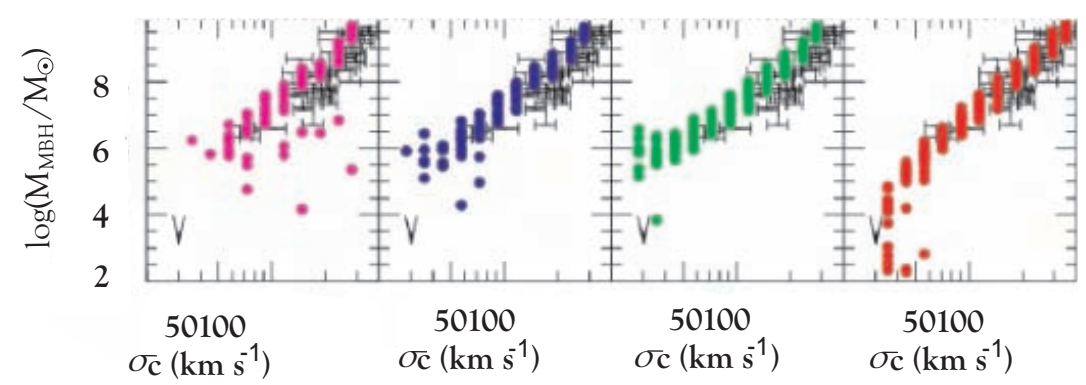

Gráficos $\mathrm{M}_{\mathrm{bh}}-\sigma_{\mathrm{c}}$ para cuatro modelos de formación de ANSs. Los tres primeros (magenta, azul y verde) son propuestos por Volonteri et al. (2008), y se basan en fusiones jerárquicas (en árbol) de halos de materia oscura. El cuarto (rojo) es propuesto por otros autores y considera que los ANSs se forman a partir de otros más pequeños, remanentes del colapso de estrellas de Población III. Las barras de error se toman de mediciones reales para $\mathrm{M}_{\mathrm{bh}} \mathrm{y} \sigma \overline{\mathrm{c}}$ 


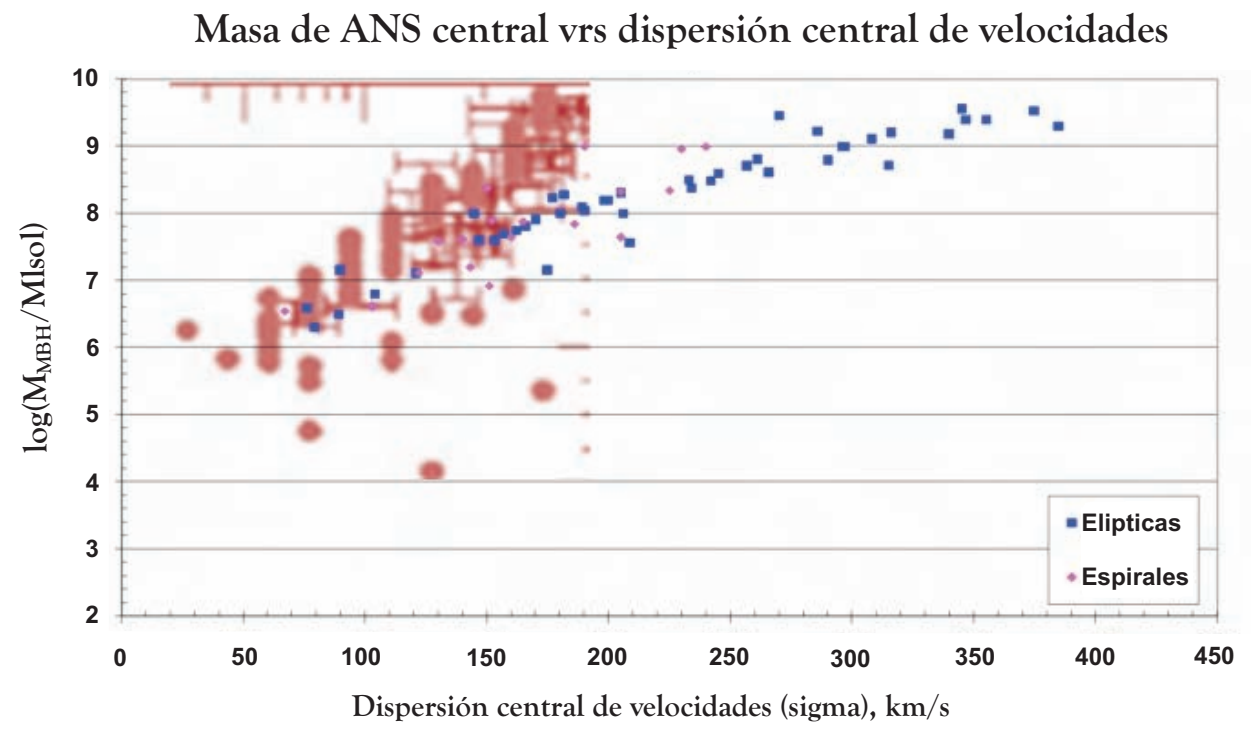

Primer modelo (color café) contra los datos recopilados en este trabajo. Considera la formación de ANSs con masas de $10^{4}$ a $10^{9}$ masas solares

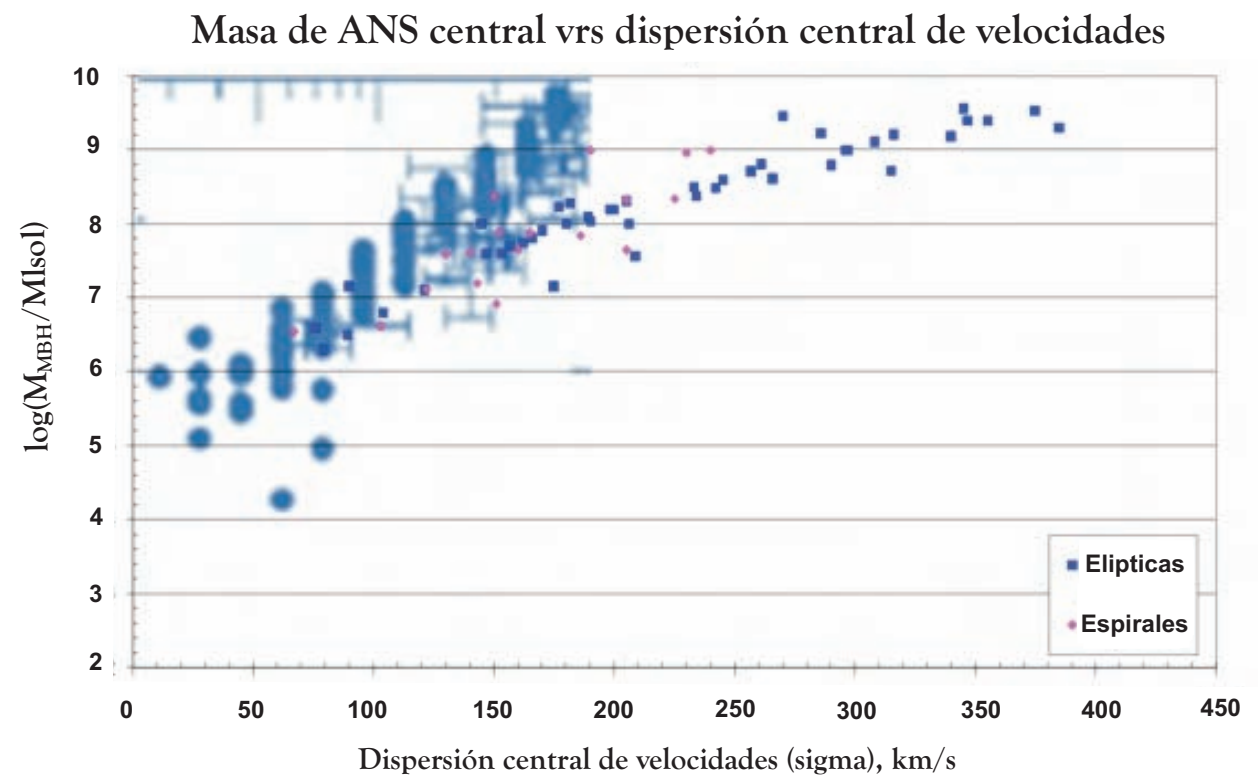

Segundo modelo (color azul) contra los datos recopilados en este trabajo. Considera la formación de ANSs con masas de $10^{4}$ a $10^{9}$ masas solares. 


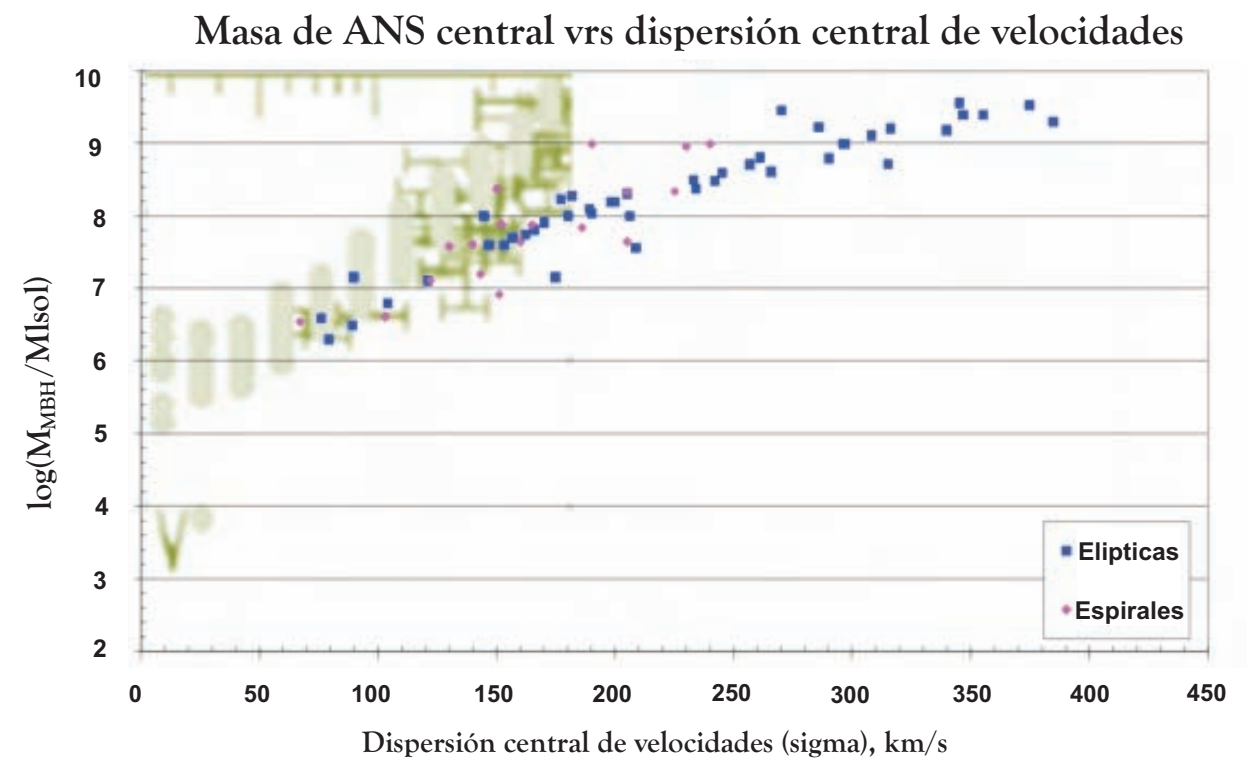

Tercer modelo (color verde) contra los datos recopilados. Predice la formación de ANSs con masas de $10^{5}$ a $10^{9}$ masas solares. Se ajusta mejor a los datos recopilados, con masas de $10^{6}$ a $10^{9}$ masas solares.

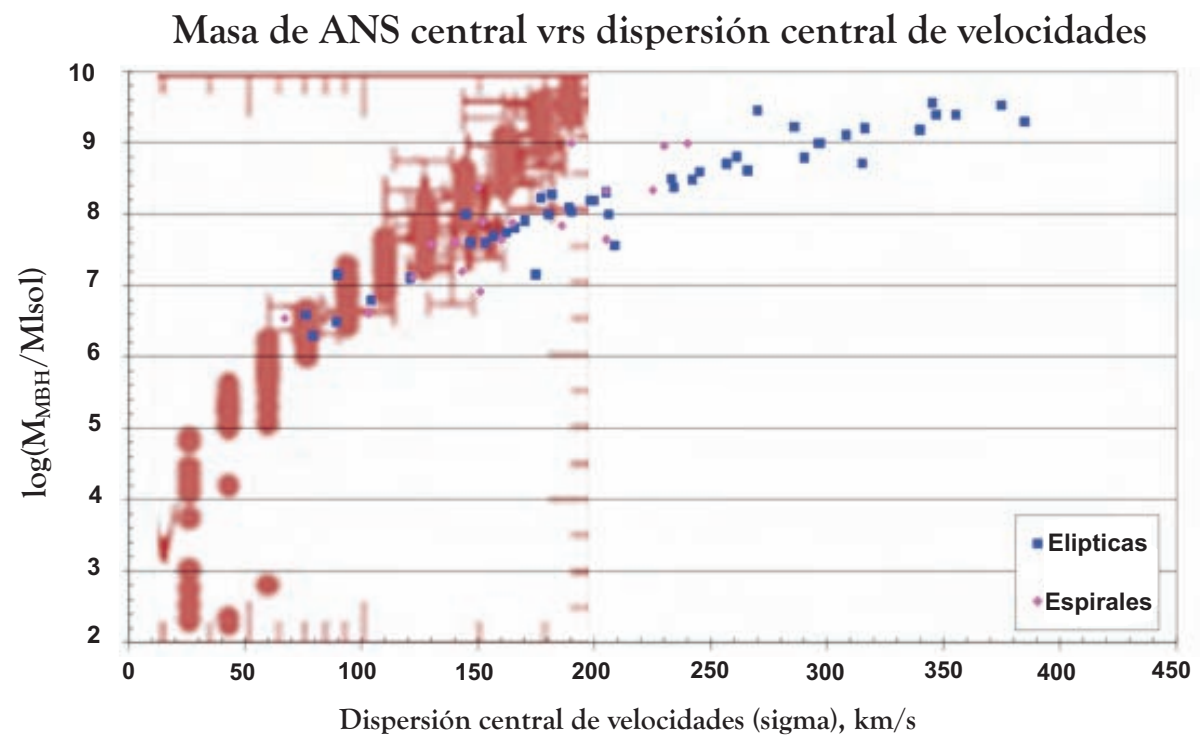

Cuarto modelo (color rojo) contra los datos recopilados en este trabajo. Considera la formación de ANSs desde $10^{2}$ masas solares. 


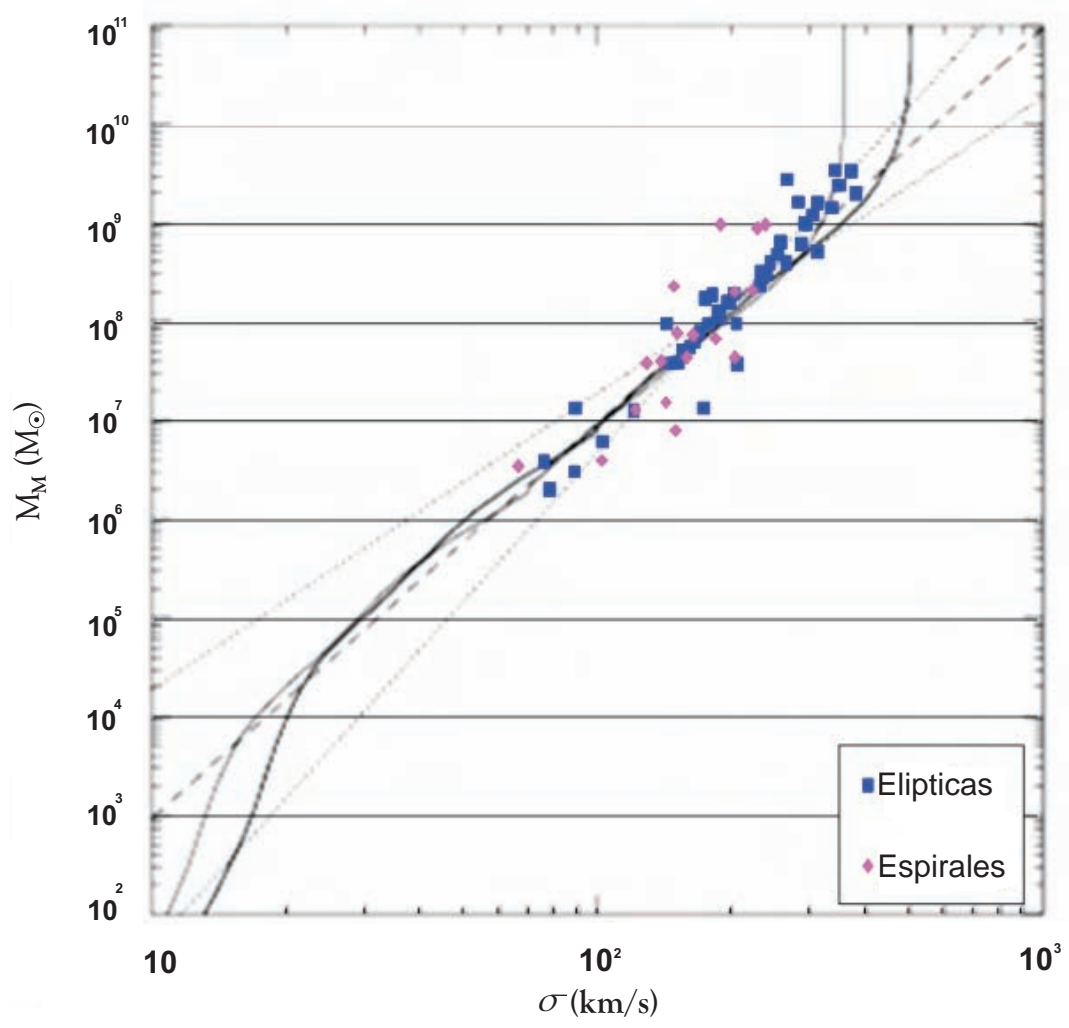

Las curvas mostradas en la diapositiva anterior son distintos tipos de modelos de la correlación $\mathrm{M}_{\mathrm{bh}}$ c generadas por Ciotti (2008), para galaxias elípticas.

Nótese que puntos azules correspondientes a galaxias elípticas son los que mejor se ajustan a la curva sólida fina, que se caracteriza porque la relación entre la masa de gas que es acretada por el agujero negro y la masa de las estrellas es $\mathrm{M}_{\text {gas }} / \mathrm{M}_{\text {star }}=$ 0.0015 , lo que significa que las tasas de acreción de ese modelo no son ni muy altas ni muy bajas, sino más bien intermedias.

\section{CONCLUSIONES}

Se ha observado una característica que se repite en varios modelos: las tasas de acreción de agujeros negros en unos modelos y las tasas de formación de agujeros negros semilla en otros modelos no son ni muy altas ni muy bajas, sino intermedias.

En los modelos de Volonteri-Lodato-Natarajan se parte de halos de materia oscura inicial a $z=20$, cada uno con un disco proto-galáctico de gas de estabilidad 
intermedia, en el que se forman agujeros negros semilla por acreción de los discos de gas, con eficiencia de formación intermedia. Cuando cesa la formación de agujeros negros semilla, la población evoluciona de acuerdo a un "escenario dirigido por fusiones" de los halos de materia oscura. Nótese que los tres modelos mencionados están sesgados hasta sigma $=200 \mathrm{~km} / \mathrm{s}$. Los datos recopilados que se ajustan mejor a estos modelos son los datos de las galaxias de disco con dispersiones de velocidades y masas más bajas que las galaxias elípticas. Esto apoya la propuesta de muchos autores, de que las primeras galaxias en formarse serían galaxias de disco.

En el modelo de Ciotti (2008) la curva masa de agujero negro dispersión central de velocidades $\left(\mathrm{M}_{\mathrm{BH}}{ }^{-}\right)$, los datos recopilados para galaxias elípticas coinciden mejor con la curva sólida fina del modelo, que los datos para galaxias de disco. Esta curva se caracteriza porque el cociente de la masa de gas acretado por el agujero negro central entre la masa total de estrellas de la galaxia es bajo $\left(\mathrm{M}_{\text {gas }} \mathrm{M}_{\text {total }}=0.0015\right)$, lo que significa que la tasa de crecimiento por acreción del ANS es más bien intermedia que baja o alta. Este modelo se ajusta mejor a valores mayores de dispersión de velocidades y de masa, lo que podría apoyar la idea de que las últimas galaxias en formarse serían las elípticas, por sus valores de masa más grandes.

Ya sea que los agujeros negros se hayan formado partiendo de agujeros negros semilla, en discos de gas, dentro de halos de materia oscura, a eficiencias intermedias, o hayan crecido por acreción de material a tasas moderadas, todo parece indicar que los procesos de crecimiento de ANSs fueron más bien estables que violentos, al menos en sus comienzos.

Dado que las correlaciones Mbh-sigma de Volonteri coinciden más con los datos recopilados para galaxias de disco, a valores de sigma menores que $200 \mathrm{~km} / \mathrm{s}$, es probable que las galaxias de disco hayan sido las primeras en formarse, alrededor de agujeros negros semillas, que luego crecerían mediante fusiones sucesivas de sus halos anfitriones.

Dado que el modelo de Ciotti se ajusta mejor a galaxias elípticas, con valores más altos de masa y de sigma, es posible que estas galaxias se hayan formado a raíz de fusiones sucesivas de las primeras.

\section{AGRADECIMIENTOS}

Agradecemos a la Dirección de Investigación Científica por la beca básica de 
investigación No 02-BBI-2007, que fue crucial para la realización de este trabajo; a la Prof. María Cristina Pineda de Carías, Directora del Observatorio Astronómico Centroamericano de Suyapa, por su constante esfuerzo para completar esta investigación, y su apoyo en la aprobación del mismo; al Dr. José Funes, por su orientación como tutor de tesis, base para la realización de este trabajo, y por sus valiosos comentarios en la revisión de este trabajo; a la Dra. Silvia Fernández, de la Universidad de Córdoba, Argentina, también por su contribución en la revisión del trabajo; a la Lic. Leticia Salomón, al Ing. Alberto Urbina, a la Lic. Margarita Arriaga ya todo el personal de la DICU durante el seguimiento de este proyecto.

\section{BIBLIOGRAFÍA}

Castillo Rosales, Y. S. Agujeros Negros Súper Masivos en el Centro de Galaxias de Disco y su Relación con Propiedades Globales de las Galaxias que los Albergan. [Tesis de Maestría] Tegucigalpa: UNAH. 2005.

Ciotti, L. "Co-evolution of elliptical galaxies and their central black holes. Clues from their scaling laws". La Rivista del Nuovo Cimento. 31 (8): 50-51. 2008.

Dudik, R. P., Satyapal, S., Gliozzi, M. \& Sambruna, R. M. "A Chandra Snapshot Survey of Infrared-bright LINERs: A Possible Link Between Star Formation, Active Galactic Nucleus Fueling, and Mass Accretion". The Astrophysical Journal. 620 (1): 113-125. 2005.

Ferrarese, L. "Black Hole Demographics". Physics World. 15 (6): 41-46. 2002. Ferrarese, L. "Supermassive Black Hole Research in the Post-HST Era". ASP (Astronomical Society of Pacific) Conference Proceedings. 291: 196. 2003.

Ferrarese, L., \& Merrit, D. "Supermassive Black Holes". Revista Physics World. 15 (6): 41-46. 2002.

Ferrarese, L., \& Merrit, D. "A Fundamental Relation between Supermassive Black Holes and Their Host Galaxies". Astrophysical Journal Letters. 539: L9-L12. 2000.

Ferrarese, L., Pogge, R. W., Peterson, B. M., Merritt, D., Wandel, A., \& Joseph, C. L. "Supermassive Black Holes in Active Galactic Nuclei. I. The Consistency of Black Hole Masses in Quiescent and Active Galaxies". Astrophysical Journal. 555: L79. 2001.

Gallo, E., Treu, T., Jacob, J., Woo, J-H., Marshall, P. J. \& Antonucci, R. "AMUSEVirgo I. Super-massive black holes in low-mass spheroids". Astrophysical Journal. 680 (1): 154-168. 2008.

Gebhardt, K., Kormendy, J., Ho, L. C., Bender, R., Bower, G., Dressler, A., et al. "Black Hole Mass Estimates from Reverberation Mapping and from Spatially Resolved Kinematics". Astrophysical Journal Letters. 543 (1): L5L8. 2000. 
Häring, N., \& Rix, H. W. "On the Black Hole Mass-Bulge Mass Relation". The Astrophysical Journal. 604 (2): L89-L92. 2004.

Ho, L. C., Filippenko, A. V., \& Sargent, W. L. "A Search for " Dwarf" Seyfert Nuclei. III. Spectroscopic Parameters and Properties of the Host Galaxies". Astrophysical Journal Supplement. 112: 315. 1997.

Marconi, A. \& Hunt, L. K. "The Relation between Black Hole Mass, Bulge Mass, and Near-Infrared Luminosity". Astrophysical Journal. 589 (1): L21-L24. 2003.

Satyapal, S., Sambruna, R. M. \& Dudik, R. P. "Ajoint mid-infrared spectroscopic and $X$-ray imaging investigation of LINER galaxies". Revista Astronomy \& Astrophysics. 414: 825-838. 2004.

Shields, G. A., Gebhardt, K., Salviander, S., Wills, B. J., Xie, B., Brotherton, M. S., et al. "The Black Hole-Bulge Relationship in Quasars". Astrophysical Journal. 583 (1): 124-133. 2003.

Vestergaard, M. "Early growth and efficient accretion of massive black holes at high redshiff'. The Astrophysical Journal. 601 (2): 676-691. 2004.

Volonteri, M., Lodato, G., \& Natarajan, P. "The evolution of massive black hole seeds". Revista Montly Notices of the Royal Astronomical Society (MNRAS). 383 (3): 1079-1088. 2007.

Wu, Q., \& Cao, X. "Origin of Radio Emission from Nearby Low-Luminosity Active Galactic Nuclei". Astrophysical Journal. 621 (1): 130-138. 2005.

Yu, Q., \& Lu, Y. "Constraints on QSO Models from a Relation between the QSO Luminosity Function and the Local Black Hole Mass Function". The Astrophysical Journal. 602 (2): 603-624. 2004 